\title{
QCD Plasma Instabilities and Bottom-Up Thermalization
}

\author{
Peter Arnold and Jonathan Lenaghan \\ Department of Physics, University of Virginia, \\ P.O. Box 400714 Charlottesville, VA 22901-4714 \\ Guy D. Moore \\ Department of Physics, McGill University, \\ 3600 University St., Montréal, QC H3A 2T8, Canada
}

(Dated: February 2003)

\begin{abstract}
We study the role of QCD plasma instabilities in non-equilibrium quark-gluon plasmas. First, we argue that such instabilities must drastically modify the "bottom-up" thermalization scenario for heavy-ion collisions. Second, we discuss conditions for the existence of instabilities in a more general context than previously treated in the QCD literature. We also give a thorough qualitative review of the origin of instabilities. We discuss some mechanisms whereby the growth of plasma instabilities saturates. Finally, we solve explicitly for instabilities and their growth rates for two extreme cases of anisotropic non-equilibrium plasmas that can be treated relatively simply and analytically: $f(\boldsymbol{p})=F\left(p_{\perp}\right) \delta\left(p_{z}\right)$ and $f(\boldsymbol{p})=F\left(p_{z}\right) \delta^{(2)}\left(p_{\perp}\right)$, where $f(\boldsymbol{p})$ is the distribution of particles in momentum space.
\end{abstract}




\section{INTRODUCTION}

Mrówczyński [1-6] has long advocated that plasma instabilities may play an important role in the thermalization of quark-gluon plasmas from anisotropic, non-equilibrium initial conditions, such as those pertaining to heavy-ion collisions. (For other early discussions, see Refs. [7-11].) Since heavy-ion collisions are rather complicated, with a large variety of different physical processes playing roles at different stages of the collision, it is useful to examine this proposal in a theoretically clean limit. A natural framework for such an investigation has been provided by Baier, Mueller, Schiff, and Son [12]. In a beautiful paper, they consider what happens shortly after the collision in the saturation scenario [1316], at energies high enough that the effective strong coupling $\alpha$ at the relevant momentum scale $Q_{\mathrm{s}}$ (known as the saturation scale) is weak, $\alpha\left(Q_{\mathrm{s}}\right) \ll 1$. The result of their analysis, which found an interesting variety of parametrically different time scales characteristic of different stages of thermalization ( $\operatorname{such}$ as $\alpha^{-3 / 2} Q_{\mathrm{s}}^{-1}, \alpha^{-5 / 2} Q_{\mathrm{s}}^{-1}$, and $\alpha^{-13 / 5} Q_{\mathrm{s}}^{-1}$ ), is known as bottom-up thermalization. We will show that even the very first moments of this scenario are drastically modified by the effects of plasma instabilities, which were not considered by Baier et al.

We will use collisionless kinetic theory coupled to soft classical gauge fields (also known as the Hard Thermal Loop approximation) to study the plasma instabilities during the first phase of bottom-up thermalization. First, in section I A, we will briefly review the first phase of the bottom-up thermalization scenario and summarize our argument that it must be drastically modified. We also argue that the collisionless approximation is appropriate to check for instabilities. For those readers more interested in the plasma instabilities of kinetic theory than in the details of the bottom-up thermalization scenario, the upshot is that we will be motivated to study the existence and properties of plasma instabilities for an initial, homogeneous, non-equilibrium, phase-space distribution of hard gluons of approximately the following form:

$$
f(\boldsymbol{p}, \boldsymbol{x})=F\left(p_{\perp}\right) \delta\left(p_{z}\right),
$$

where $p_{\perp} \equiv \sqrt{p_{x}^{2}+p_{y}^{2}}$. This is an interesting limiting case of certain classes of distributions that have been studied in the literature numerically by Randrup and Mrówczyński [6] and Romatschke and Strickland [17], but (1.1) has the advantage of being more analytically tractable. ${ }^{1}$ The particular plasma instabilities that will be of interest are known in various contexts as (i) Weibel [19], filamentation, or pinching instabilities, and (ii) two-stream or Buneman [28] instabilities, with the names somewhat interchangeable in each category. Because many readers will not be intimately familiar with these instabilities in kinetic theory, we will review them both formally and qualitatively in section II for parity-invariant situations. Typically, for parity-invariant particle momentum distributions, plasma instabilities exist (in the collisionless approximation) whenever those distributions are anisotropic. We will give conditions which make this statement precise. We also discuss certain aspects of the physics that eventually cuts off the growth of such instabilities and point out the qualitative differences between the cases of Abelian and non-Abelian gauge theories. To make the rather

\footnotetext{
${ }^{1}$ Romatschke and Strickland [17] do obtain analytic results in a more general class of situations, but their results are unwieldy enough that they refrain from giving them explicitly in their paper. Also, another example of analysis of instabilities in ultra-relativistic plasmas, generalized to inclusion of a background magnetic field but restricted to wave vectors in certain symmetry directions, may be found in Ref. [18].
} 


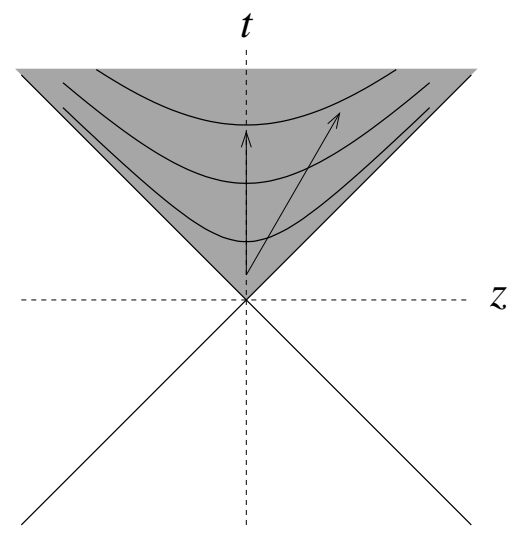

FIG. 1: The standard picture of lines of constant proper time $\tau=\sqrt{t^{2}-z^{2}}$ in a high-energy collision, in which the $45^{\circ}$ lines are the colliding nuclei. The two arrows show how free-streaming partons leaving from the same initial point would later segregate themselves in $z$ according to their value of $v_{z}$.

general discussion in section II more concrete, we will then analyze in detail in section III the instabilities associated with the particle distribution (1.1). This distribution is simple enough that much of the analysis can be done analytically. We also give a specific example in section IV of how non-Abelian interactions can cut off the growth of plasma instabilities. Various matters are left for appendices, including an analytic analysis of instabilities for the distribution

$$
f(\boldsymbol{p}, \boldsymbol{x})=F\left(p_{z}\right) \delta^{(2)}\left(\boldsymbol{p}_{\perp}\right),
$$

which is complementary to that of (1.1).

We will show that plasma instabilities play a crucial role in the post-saturation evolution of heavy-ion collisions, in the framework originally established for bottom-up thermalization, but we are not yet able to give detailed parametric estimates of the time scale for equilibration (analogous to the $\alpha^{-13 / 5} Q_{\mathrm{s}}^{-1}$ time scale of Baier et al.). We do expect thermalization to be more rapid, but this is a topic for future research.

\section{A. The first phase of bottom-up thermalization}

Suppose the initial nuclei are moving in the $\pm z$ directions. The starting point for the bottom-up thermalization scenario is to consider an initial distribution of particles that is (i) boost-invariant in the $z$ direction, (ii) homogeneous in the $x$ and $y$ directions, and (iii) dominated by gluons with momenta $p$ of order some scale $Q_{\mathrm{s}}$ and with non-perturbative occupation numbers per mode of order $1 / \alpha$. For sufficiently high energy collisions, the first assumption should be adequate for describing the central rapidity region and the second assumption for describing local thermalization for large nuclei. These two assumptions imply that physical quantities (measured in a local rest frame) depend only on the proper time $\tau=\sqrt{t^{2}-z^{2}}$, as depicted in Fig. 1. The third assumption is the saturation assumption and is taken to hold at $\tau \sim Q_{\mathrm{s}}^{-1}$. The bottom-up thermalization scenario concerns itself with what happens at later times $\tau \gg Q_{\mathrm{s}}^{-1}$, given this initial condition. Following Baier et 
al., we will refer to the initial gluons as "hard" gluons. ${ }^{2}$

If there were no interactions, then, as time progressed, the hard gluons would simply follow straight-line trajectories and segregate themselves in $z$ according to their values of $v_{z}=p_{z} / p$, as depicted in Fig. 1. One can show that, in a local rest frame (where the average $\boldsymbol{p}$ vanishes), the resulting distribution of hard gluons for $\tau \gg Q_{\mathrm{s}}^{-1}$ would then be dominated by gluons with $p_{x} \sim p_{y} \sim Q_{\mathrm{s}}$ but $p_{z} \sim 1 / \tau \ll Q_{\mathrm{s}}$. The occupation numbers of these modes would still be of order $1 / \alpha$. The spatial number density $N_{\mathrm{h}}$ of hard gluons decreases linearly with the linear expansion as

$$
N_{\mathrm{h}} \sim \frac{Q_{\mathrm{s}}^{3}}{\alpha\left(Q_{\mathrm{s}} \tau\right)}
$$

Baier et al. argue that small-angle scattering between the hard gluons instead widens the spread in $p_{z}$ to

$$
p_{z} \sim \frac{Q_{\mathrm{s}}}{\left(Q_{\mathrm{s}} \tau\right)^{1 / 3}}
$$

during the first stage, $1 \ll Q_{\mathrm{s}} \tau \ll \alpha^{-3 / 2}$, of their scenario. They then argue that hard gluon number is conserved over the short duration of this first stage, and so the spatial number density of hard gluons still decreases linearly with $\tau$ as (1.3). The occupation number per mode (related by $N_{\mathrm{h}}=\int_{\boldsymbol{p}} f_{\mathrm{h}} \sim p_{x} p_{y} p_{z} f_{\mathrm{h}}$ ) then decreases as

$$
f_{\mathrm{h}} \sim \frac{N_{\mathrm{h}}}{Q_{\mathrm{s}}^{2} p_{z}} \sim \frac{1}{\alpha\left(Q_{\mathrm{s}} \tau\right)^{2 / 3}}
$$

The fact that $f_{\mathrm{h}} \ll 1 / \alpha$ for $Q_{\mathrm{s}} \tau \gg 1$ means that the direct interactions between hard particles can be treated perturbatively at these times.

Unscreened Coulomb scattering is infrared divergent. The small-angle scattering of hard gluons discussed above is therefore dominated by momentum exchanges of order the inverse screening length. Baier et al. estimated this soft scale, which we will call $m_{\text {soft }}$, to be ${ }^{3}$

$$
m_{\mathrm{soft}}^{2} \sim \alpha \int_{\boldsymbol{p}} \frac{f_{\mathrm{h}}(\boldsymbol{p})}{p} \sim \frac{\alpha N_{\mathrm{h}}}{Q_{\mathrm{s}}} \sim \frac{Q_{\mathrm{s}}}{\tau}
$$

They loosely referred to $m_{\text {soft }}$ as the Debye mass $m_{\mathrm{D}}$ and took estimates from the literature [20-22]. In fact, the Debye mass is not precisely the correct quantity to use since one really wants to use the full self-energy $\Pi^{\mu \nu}(\omega, \boldsymbol{q})$ for the soft exchanged gluon of the small-angle scattering process. Also, the Debye mass is not precisely defined in anisotropic situations: it depends on direction. However, these details do not affect parametric estimates, and so (1.6) is a perfectly adequate estimate of the relevant soft physics scale. There is only one problem. A parametric estimate does not necessarily reveal the sign of the result. As we shall see, a more appropriate definition of $m_{\text {soft }}^{2}$ reveals that its sign is negative for certain modes,

${ }^{2}$ This usage is to distinguish them from lower energy partons generated later in the evolution of the collision. From the point of view of the colored glass condensate picture of the nucleus, or of "mini-jet" analyses of heavy ion collisions, the usage may seem peculiar, since $Q_{\mathrm{s}}$ is treated as an infrared cutoff in those contexts.

3 Our terminology is slightly different from that of Baier et al. [12]. They refer to (1.4) as the "soft" physics scale during the first stage of their scenario. In our paper, the term "soft" will instead generally refer to (1.6). 
corresponding to an instability in the soft gauge fields in the presence of the anisotropic distribution of hard gluons. These unstable soft modes then grow exponentially with a characteristic time scale of

$$
t_{\text {growth }} \sim \frac{1}{\left|m_{\text {soft }}\right|} \sim \sqrt{\frac{\tau}{Q_{\mathrm{s}}}} .
$$

Because the growth time $t_{\text {growth }}$ is parametrically small compared to the expansion time $\tau$ for $Q_{\mathrm{s}} \tau \gg 1$, the first stage of bottom-up thermalization is drastically modified by the generation of large, non-perturbative soft gauge fields. Since this is true for any $Q_{\mathrm{s}} \tau \gg 1$, the first stage of the original bottom-up thermalization scenario cannot, even briefly, be qualitatively accurate.

In order to demonstrate the instability, note that the first stage of the bottom-up scenario is described by hard gluons with $p_{z} \ll p_{x} \sim p_{y} \sim Q_{\mathrm{s}}$ as in (1.4). Using the azimuthal symmetry of the problem, such a distribution of hard gluons can be approximately described as having the form (1.1). In Sec. III, we will analyze the soft instabilities created by hard distributions of this form.

The extreme planarity (oblateness) of the anisotropy of our model distribution (1.1) is somewhat an oversimplification of the actual situation in the bottom-up scenario. Baier et al. point out that not only hard gluons contribute to $m_{\mathrm{soft}}^{2}$, but so also do softer gluons which have been generated by Bremsstrahlung and then pushed to momenta $k_{\mathrm{s}}$ of order $p_{z}(1.4)$ by scattering from hard particles. These softer gluons will be anisotropic but not have the extreme anisotropy of the hard gluons. In the bottom-up thermalization scenario, Baier et al. show that such soft gluons give a contribution to $m_{\text {soft }}^{2}$ comparable to that of the hard gluons. We will see that whenever there is significant deviation from anisotropy, then there are unstable modes with momenta of order $m_{\text {soft }}$, and so plasma instabilities will indeed modify the bottom-up scenario. However, as a simple analytically-tractable example of this phenomena, it will be instructive to ignore the softer contribution and study the effects of just the extreme oblate distribution (1.1) of the hard gluons.

In our analysis of plasma instabilities and their growth, we will use a collisionless approximation for the hard particles. We need to justify this approximation since a significant rate of collisions could eliminate the instability. Baier et al. estimate the rate of hard particle collisions for a single hard gluon in the first stage of their scenario as

$$
\frac{d N_{\mathrm{col}}}{d \tau} \sim \sigma N_{\mathrm{h}}\left(1+f_{\mathrm{h}}\right) \sim \frac{\alpha N_{\mathrm{h}}}{m_{\mathrm{soft}}^{2} p_{z} \tau},
$$

where $\sigma \sim \alpha^{2} m_{\text {soft }}^{2}$ is the cross-section. Using the previous estimates for $N_{\mathrm{h}}, m_{\mathrm{soft}}$, and $p_{z}$, the mean free time between such collisions is then

$$
t_{\mathrm{col}} \sim\left(\frac{d N_{\mathrm{col}}}{d \tau}\right)^{-1} \sim \frac{\left(Q_{\mathrm{s}} \tau\right)^{2 / 3}}{Q_{\mathrm{s}}} .
$$

For $Q_{\mathrm{s}} \tau \gg 1$, this is parametrically long compared to the time scale $t_{\text {growth }}$ of (1.7) for the growth of instabilities. We may therefore ignore collisions of hard gluons for the purpose of understanding whether the bottom-up thermalization scenario is affected by plasma instabilities.

In our analysis of instabilities, we will assume that the plasma is homogeneous. In fact, from Fig. 1, one can see that densities will vary in a constant $t$ slice over a distance scale of order $\tau$. The homogeneity assumption is valid for $Q_{\mathrm{s}} \tau \gg 1$ because this scale will be large compared to the typical wavelength $1 /\left|m_{\text {soft }}\right|$ of unstable modes. 


\section{PLASMA INSTABILITIES}

The basic starting point is the expression for the soft gauge-field self-energy $\Pi^{\mu \nu}(\omega, \boldsymbol{q})$ in the presence of a given homogeneous but non-equilibrium distribution $f(\boldsymbol{p})$ of hard particles. In this section, we will treat general distributions $f(\boldsymbol{p})$ and will not yet specialize to specific forms such as the planar distribution of (1.1).

\section{A. Review of the retarded self-energy}

First, let us briefly review the known result for the self-energy from collisionless kinetic theory. In order to retain contact with the literature on non-relativistic plasmas, we will avoid specializing to the ultra-relativistic limit for the moment. For simplicity, we start by discussing Abelian gauge theories such as electromagnetism. One starts with the Vlasov equations, which are the collisionless Boltzmann equation for a hard particle distribution $f(\boldsymbol{p}, \boldsymbol{x}, t)$ and Maxwell's equations for the soft gauge fields:

$$
\begin{gathered}
\partial_{t} f+\boldsymbol{v} \cdot \nabla_{\boldsymbol{x}} f+e(\boldsymbol{E}+\boldsymbol{v} \times \boldsymbol{B}) \cdot \boldsymbol{\nabla}_{\boldsymbol{p}} f=0, \\
\partial_{\nu} F^{\mu \nu}=j^{\mu}=e \int_{\boldsymbol{p}} v^{\mu} f
\end{gathered}
$$

where $e(\boldsymbol{E}+\boldsymbol{v} \times \boldsymbol{B})$ is the Lorentz force on hard particles of charge $e, \boldsymbol{v}=\boldsymbol{v}(\boldsymbol{p})$ is the hard particle velocity for a given momentum $\boldsymbol{p}$, and $v^{\mu} \equiv(1, \boldsymbol{v})$. There is also an implicit sum over particle species and their charges on the right-hand side of (2.2). In the absence of external fields, one treats $\boldsymbol{E}(\boldsymbol{x}, t)$ and $\boldsymbol{B}(\boldsymbol{x}, t)$ as small and linearizes $f(\boldsymbol{p}, \boldsymbol{x}, t)=f_{0}(\boldsymbol{p})+f_{1}(\boldsymbol{p}, \boldsymbol{x}, t)$ in small fluctuations $f_{1}$ about some homogeneous distribution $f_{0}(\boldsymbol{p})$. We will assume that $e \int_{\boldsymbol{p}} v^{\mu} f_{0}$ vanishes (when implicitly summed over species) so that $f_{0}$ carries no net charge or net current. Then

$$
\begin{gathered}
\partial_{t} f_{1}+\boldsymbol{v} \cdot \nabla_{\boldsymbol{x}} f_{1}+e(\boldsymbol{E}+\boldsymbol{v} \times \boldsymbol{B}) \cdot \nabla_{\boldsymbol{p}} f_{0} \simeq 0 \\
\partial_{\nu} F^{\mu \nu}=e \int_{\boldsymbol{p}} v^{\mu} f_{1},
\end{gathered}
$$

which are known as linearized Vlasov equations. Fourier transforming from $(t, \boldsymbol{x})$ to $(\omega, \boldsymbol{q})$, one may solve for $f_{1}$ and obtain

$$
i Q_{\nu} F^{\mu \nu} \simeq i e^{2} \int_{\boldsymbol{p}} \frac{v^{\mu}(\boldsymbol{E}+\boldsymbol{v} \times \boldsymbol{B}) \cdot \boldsymbol{\nabla}_{\boldsymbol{p}} f_{0}}{-\omega+\boldsymbol{v} \cdot \boldsymbol{q}-i \epsilon}
$$

where the $\epsilon$ is a positive infinitesimal inserted as a prescription to obtain the retarded solution. We use the shorthand notation

$$
\int_{\boldsymbol{p}} \cdots \equiv \int \frac{d^{3} p}{(2 \pi)^{3}} \cdots
$$

We may rewrite $(2.5)$ as

$$
i Q_{\nu} F^{\mu \nu} \simeq-\Pi^{\mu \nu} A_{\nu}
$$

extracting the result for the retarded self-energy $\Pi^{\mu \nu}$,

$$
\Pi^{\mu \nu}(\omega, \boldsymbol{q})=e^{2} \int_{\boldsymbol{p}} \frac{\partial f(\boldsymbol{p})}{\partial p^{k}}\left[-v^{\mu} g^{k \nu}+\frac{v^{\mu} v^{\nu} q^{k}}{-\omega+\boldsymbol{v} \cdot \boldsymbol{q}-i \epsilon}\right]
$$


where we use $(-+++)$ metric convention, and Roman indices such as $k$ are spatial indices that run from 1 to 3 . The self-energy $\Pi^{\mu \nu}$ is symmetric in the indices $\mu \nu .{ }^{4}$ It is also useful to note that the above expression satisfies the Ward identity, $Q_{\mu} \Pi^{\mu \nu}=0$ (which is just current conservation, $\left.Q_{\mu} j^{\mu}=0\right)$, where $Q \equiv(\omega, \boldsymbol{q})$. This implies

$$
\Pi^{0 \nu}=\frac{q^{i} \Pi^{i \nu}}{\omega}, \quad \Pi^{00}=\frac{q^{i} \Pi^{i j} q^{j}}{\omega^{2}} .
$$

The result for non-Abelian gauge theory ${ }^{5}$ is essentially identical except that $e^{2}$ is replaced by $g^{2} \operatorname{tr}\left(T_{s}^{a} T_{s}^{b}\right)=\delta^{a b} C_{s} d_{s} / d_{\mathrm{A}}$ for each species $s$ of hard particle, where $g$ is the gauge coupling, $a$ and $b$ are adjoint color indices for the soft gluon, $T_{s}^{a}$ are the color generators for the hard particle's color representation, $C_{s}$ is the quadratic Casimir given by $T_{s}^{a} T_{s}^{a}=C_{s}$, and $d_{s}, d_{\mathrm{A}}$ are the dimensions of the particle's representation and the adjoint representation respectively. For gluons in $\mathrm{QCD}, C_{s} d_{s} / d_{\mathrm{A}}=3$, while for quarks $C_{s} d_{s} / d_{\mathrm{A}}=1 / 2$. However, since there is no essential difference between the Abelian and non-Abelian results of the linearized analysis, we will generally write the self-energy as (2.8) for the sake of brevity, with the understanding that

$$
e^{2} \rightarrow \sum_{s} \nu_{s} \frac{g^{2} C_{s} d_{s}}{d_{\mathrm{A}}}
$$

with $f \rightarrow f_{s}$, where $\nu_{s}$ represents the number of degrees of freedom of a given hard particle species, excluding color. (So, for instance, if $f_{\mathrm{g}}$ for gluons represents the density of gluons per spin state and color, then $\nu_{\mathrm{g}}$ would be 2.)

Isotropic distributions $f(\boldsymbol{p})=f(p)$ have the following special properties: (i) $\Pi(0, \boldsymbol{q})$ is diagonal with the only non-zero entry $\Pi^{00}(0, \boldsymbol{q})=-m_{\mathrm{D}}^{2}$, where $m_{\mathrm{D}}$ is the Debye screening mass (inverse Debye screening length); and (ii) $\Pi(\omega, 0)$ is diagonal with the only non-zero entries $\Pi^{i j}(\omega, 0)=m_{\mathrm{pl}}^{2} \delta^{i j}$, where $m_{\mathrm{pl}}=m_{\mathrm{D}} / \sqrt{3}$ is the plasmon mass (plasma frequency). In contrast, for generic anisotropic distributions, (i) $\Pi(0, \boldsymbol{q})$ is not diagonal and depends on the direction $\hat{\boldsymbol{q}}$, and (ii) $\Pi(\omega, 0)$ is not diagonal and the eigenvalues of its spatial part are not all the same.

\section{B. Criteria for Instability}

In momentum space $Q=(\omega, \boldsymbol{q})$, the linearized effective equation (2.7) for the soft gauge fields is

$$
\left[\left(-\omega^{2}+q^{2}\right) g^{\mu \nu}-Q^{\mu} Q^{\nu}+\Pi^{\mu \nu}(\omega, \boldsymbol{q})\right] A_{\nu}=0 .
$$

For a given $\boldsymbol{q}$, there will be instabilities if there are any solutions for $\omega$ with $\operatorname{Im} \omega>0$.

For the case of parity-invariant background particle distributions, $f(\boldsymbol{p})=f(-\boldsymbol{p})$, there are some simple sufficient conditions for the existence of instabilities. In the rest of this paper, we will assume that distributions are parity invariant. ${ }^{6}$ There is then a simple sufficient (but not necessary) condition for the existence of an instability:

\footnotetext{
${ }^{4}$ The symmetry is not obvious for the first term in (2.8) unless one integrates by parts and uses $\boldsymbol{v}=\boldsymbol{\nabla}_{\boldsymbol{p}} E_{\boldsymbol{p}}$ so that $\partial_{p^{j}} v^{i}=\partial_{p^{j}} \partial_{p^{i}} E_{\boldsymbol{p}}$, where $E_{\boldsymbol{p}}$ is the energy of a hard particle with momentum $\boldsymbol{p}$.

5 See Ref. [5] for the non-Abelian version of (2.8) in the non-equilibrium case. However, the basic modifications have been known in various approximations for ages (e.g. Ref. [23]). Ref. [24] also has expressions for (2.8) specialized to the case of axial symmetry.

6 We also implicitly assume that hard particle dispersion relations are parity invariant.
} 


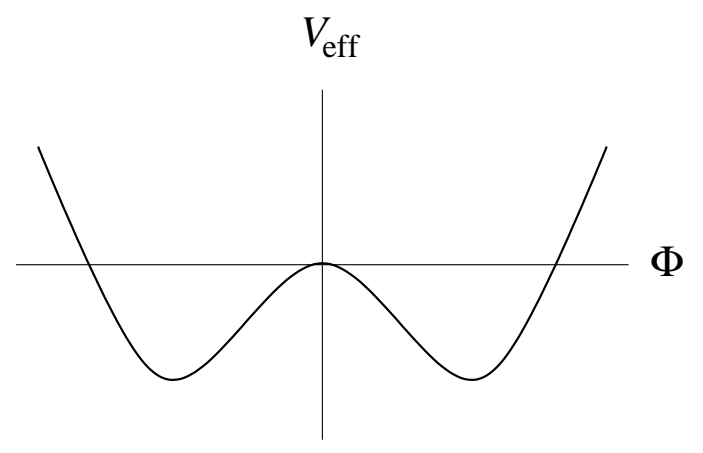

FIG. 2: Example of an effective potential $V_{\text {eff }}(\Phi)$ in scalar field theory for which $\Phi=0$ would be unstable.

Condition 1. There is an instability associated with a given wavenumber $\boldsymbol{q}$ for each negative eigenvalue of the $3 \times 3$ matrix $q^{2} \delta^{i j}+\Pi^{i j}(0, \boldsymbol{q})$.

This is a simple generalization of the Penrose criteria used by Mrówczyński in Ref. [2] for a special case. There is a simple continuity argument for this assertion, which we give in Appendix A $1 .^{7}$

The zero-frequency self-energy $\Pi(0, \boldsymbol{q})$ depends only on the direction $\hat{\boldsymbol{q}}$ and not the magnitude $q$ of $\boldsymbol{q}$ because $(2.8)$ can be rewritten for $\omega=0 \mathrm{as}^{8}$

$$
\Pi^{\mu \nu}(0, \boldsymbol{q})=e^{2} \int_{\boldsymbol{p}} \frac{\partial f(\boldsymbol{p})}{\partial p^{k}}\left[-v^{\mu} g^{k \nu}+\frac{v^{\mu} v^{\nu} \hat{q}^{k}}{\boldsymbol{v} \cdot \hat{\boldsymbol{q}}-i \epsilon}\right] .
$$

We will write $\Pi(0, \hat{\boldsymbol{q}})$ instead of $\Pi(0, \boldsymbol{q})$ to emphasize this. A simple corollary of Condition 1 is then that there will always be an instability in some mode (i.e. for small enough $q$ ) if $\Pi^{i j}(0, \hat{\boldsymbol{q}})$ has a negative eigenvalue.

Readers may find it useful to consider as an analogy the static effective potential $V_{\text {eff }}(\Phi)$ for the softest modes of a scalar field theory. If $V_{\text {eff }}(\Phi)$ has negative curvature at $\Phi=0$, as depicted in Fig. 2, then $\Phi=0$ is unstable. For scalars with no intrinsic mass (analogous to gauge bosons), this curvature is simply $\Pi(0)$. The condition $\Pi(0)<0$ implies that there is an instability, even though the precise calculation of the growth rate would require studying

${ }^{7}$ Ref. [2] considered the special case where $\hat{\boldsymbol{q}}$ points along a symmetry axis of $f(\boldsymbol{p})$ and the eigen-modes of $\Pi^{i j}$ are determined by symmetry. Textbook discussions of the Penrose criteria for similar special cases in the non-relativistic context may be found in Sec. 9.10.2 of Ref. [25] and Sec. 3.3.4 of Ref. [26]. We are unaware of a general analysis of the case where $\hat{\boldsymbol{q}}$ does not point in a symmetry direction of the problem, and so we give our own argument in the appendix. It also sets up the argument for Condition 2 below. Similarly, one often sees the Penrose criteria playing the role of necessary (as well as sufficient) conditions for instability in certain situations, such as where $\hat{\boldsymbol{q}}$ is in the $z$ direction and $f(\boldsymbol{p})$ has the form $F_{z}\left(p_{z}\right) F_{\perp}\left(p_{\perp}\right)$ with $F\left(p_{z}\right)$ a monotonic decreasing function of $\left|p_{z}\right|[2,25,26]$. We do not know how to prove necessity in the case where $\hat{\boldsymbol{q}}$ points in non-symmetry directions, for reasons described in the appendix.

${ }^{8}$ Physically, the independence of $\Pi(0, \boldsymbol{q})$ on the magnitude $q$ of the soft field wave number depends on $q$ being small compared to hard particle momenta - an approximation implicit in our use of the Vlasov equations. 
the dynamics, depending on $\Pi(\omega)$. Linearizing $\frac{1}{2}|\nabla \Phi|^{2}+V_{\text {eff }}(\Phi)$ about $\Phi=0$, one would find that the condition for a particular mode to be unstable is $q^{2}+\Pi(0, \boldsymbol{q})<0$, analogous to Condition 1. The picture of Fig. 2 also suggests that non-linearities in the interactions of the soft fields may eventually cut off the growth of the instability, a topic we will return to later.

We will refer to instabilities discovered by Condition 1 as "magnetic" instabilities because the condition itself involves the self-energy for $\boldsymbol{A}(\omega=0)$ and so involves magnetic but not electric fields. We will give a physical picture of the origin of the instability in Sec. IIC. Of course, the growth of magnetic fields implies the generation of electric fields as well, by Maxwell's equations, so the actual growing mode will not be purely magnetic.

Note that the Ward identity $q_{\mu} \Pi^{\mu \nu}=0$ implies $\hat{q}^{i} \Pi^{i j}(0, \boldsymbol{q})=0$. Therefore, the longitudinal polarization of $\boldsymbol{A}(\omega=0)$ is always associated with a zero eigenvalue of $\Pi^{i j}(0, \boldsymbol{q})$ and so will not play a role in Condition 1 . We could have phrased Condition 1 equivalently using its transverse projection:

Condition 1'. There is an instability associated with a given wavenumber $\boldsymbol{q}$ for each negative eigenvalue of the $3 \times 3$ matrix $q^{2} \delta^{i j}-q^{i} q^{j}+\Pi^{i j}(0, \boldsymbol{q})$.

In Appendix B, we expand upon an argument in Ref. [27] to use Condition 1 to show that some unstable mode always exists unless the matrix $\Pi^{i j}(0, \hat{q})$ is identically zero. We further show that this leads to a simple necessary as well as sufficient condition for magnetic instability in the ultra-relativistic limit:

Condition 1-b. Magnetic instabilities exist for a given (parity symmetric) distribution $f(\boldsymbol{p})$ if

$$
\mathcal{M}(\hat{\boldsymbol{p}}) \equiv \frac{e^{2}}{2 \pi^{2}} \int_{0}^{\infty} p^{2} d p \frac{v}{p} f(p \hat{\boldsymbol{p}})
$$

is anisotropic in $\hat{\boldsymbol{p}}$. Moreover, in the ultra-relativistic limit, this is a necessary (as well as sufficient) condition for the existence of instabilities.

We note in passing that the angular average of $\mathcal{M}(\hat{\boldsymbol{p}})$ over $\hat{\boldsymbol{p}}$ gives

$$
m_{\infty}^{2} \equiv\langle\mathcal{M}(\hat{\boldsymbol{p}})\rangle_{\hat{\boldsymbol{p}}}=e^{2} \int_{\boldsymbol{p}} \frac{v}{p} f(\boldsymbol{p})
$$

which defines a basic mass scale we will encounter repeatedly. In the ultra-relativistic case, it also turns out to be the effective mass of transverse electromagnetic waves in the large $q$ limit, with $\omega^{2} \simeq q^{2}+m_{\infty}^{2}$. This is shown in Ref. [27] and inspires the notation $m_{\infty}$, though this correspondence only holds in the ultra-relativistic case. Note that $m_{\infty}^{2}$ is independent of the direction $\hat{\boldsymbol{q}}$.

In Condition 1-b we have re-emphasized the underlying assumption of our discussion that $f(\boldsymbol{p})$ is parity symmetric. The condition is obviously incorrect without this assumption: Consider, for example, a standard equilibrium distribution boosted to an inertial frame where the average momentum does not vanish. The corresponding $f(\boldsymbol{p})$ and $\mathcal{M}(\boldsymbol{p})$ are then anisotropic and also not parity invariant, but equilibrium is stable, regardless of the inertial frame.

There is another type of instability that can occur, which can be associated with the electric potential $A^{0}$ at $\omega=0$ [which for non-zero $\omega$ is related to the longitudinal polarization of $\boldsymbol{A}$ by the Ward identity (2.9)]. In Appendix A 2, we show that another (independent) sufficient condition for the existence of an instability is 
Condition 2. There is an instability associated with a given wavenumber $\boldsymbol{q}$ if

$$
q^{2}-\Pi^{00}(0, \hat{\boldsymbol{q}})+\Pi^{0 i}(0, \hat{\boldsymbol{q}})\left[q^{2}+\Pi(0, \boldsymbol{q})\right]_{i j}^{-1} \Pi^{j 0}(0, \hat{\boldsymbol{q}})<0,
$$

where $\left[q^{2}+\Pi\right]^{-1}$ denotes the inverse of the $3 \times 3$ matrix $q^{2} \delta^{i j}+\Pi^{i j}$.

In the non-relativistic limit, $\Pi^{i 0}$ is suppressed by powers of $v / c$ compared to $\Pi^{00}$ and this condition becomes simply $q^{2}-\Pi^{00}(0, \hat{\boldsymbol{q}})<0$, which will happen for some $\boldsymbol{q}$ if $-\Pi^{00}(0, \hat{\boldsymbol{q}})<0$. [A simple mnemonic for this condition is to recall that, for isotropic distributions, $-\Pi^{00}(0, \boldsymbol{q})$ gives the squared Debye mass $m_{\mathrm{D}}^{2}$. So $-\Pi^{00}(0, \hat{q})<0$ is analogous to $m_{\mathrm{D}}^{2}$ having the wrong sign, which one might guess could indicate instability.] This electrostatic instability is routinely discussed in plasma physics texts ${ }^{9}$ and is typically called the two-stream or Buneman [28] instability. We will refer to instabilities discovered by Condition 2 as "electric" instabilities, even though the situation is a little more complicated in the relativistic case.

Because $\hat{q}^{i} \Pi^{0 i}(0, \hat{q})=0$, the $\hat{\boldsymbol{q}}$ polarization does not contribute in the last term of (2.15). One may then restrict the sums over $i$ and $j$ to directions transverse to $\boldsymbol{q}$ and similarly project $\Pi$ to a $2 \times 2$ matrix in the transverse subspace.

Were it not for the $i \epsilon$ prescription in the retarded self-energy $(2.8), \Pi^{0 i}(0, \boldsymbol{q})$ would vanish by parity $(\boldsymbol{p} \rightarrow-\boldsymbol{p})$. Using $(x-i \epsilon)^{-1}=$ P.P. $x^{-1}+i \pi \delta(x)$, where P.P. denotes principal part, parity then gives

$$
\Pi^{0 i}(0, \boldsymbol{q})=i \pi e^{2} \int_{\boldsymbol{p}} v^{i} \delta(\boldsymbol{v} \cdot \boldsymbol{q}) \boldsymbol{q} \cdot \nabla_{\boldsymbol{p}} f(\boldsymbol{p}),
$$

which is pure imaginary. We may therefore rewrite Condition 2 as

$$
q^{2}-\Pi^{00}(0, \hat{\boldsymbol{q}})-\left[\operatorname{Im} \Pi^{0 i}(0, \hat{\boldsymbol{q}})\right]\left[q^{2}+\Pi \mathbf{\Pi}(0, \hat{\boldsymbol{q}})\right]_{i j}^{-1}\left[\operatorname{Im} \Pi^{j 0}(0, \hat{\boldsymbol{q}})\right]<0 .
$$

If there are no magnetic instabilities, so that $q^{2}+\Pi(0, \boldsymbol{q})$ is positive definite, then the last term contributes negatively to the left-hand side. In this case, a weaker sufficient condition for an electric instability is then that $q^{2}-\Pi^{00}(0, \hat{\boldsymbol{q}})<0$.

Our categorization of instabilities as "magnetic" or "electric" is not always physically significant. We use this terminology to tell us whether the existence of the instability was indicated by Condition 1 or Condition 2 respectively. Condition 1 is related to the transverse eigen-values of the zero-frequency self-energy $\Pi^{i j}(0, q)$, and Condition 2 is related to the $\hat{\boldsymbol{q}}$ polarization [see Appendix A 2]. However, the actual growing unstable solutions to the dispersion relation (2.11) have non-zero frequency $\omega$ (with $\operatorname{Im} \omega>0$ ). The corresponding polarizations can look quite different from those of $\Pi(0, \boldsymbol{q})$ in generic situations where eigenpolarizations of $\Pi^{i j}(\omega, \boldsymbol{q})$ are not fixed by symmetry. In particular, we will see a specific example in Sec. III where, for certain limiting cases of $\boldsymbol{q}$, the actual growing unstable modes for a "magnetic" instability and "electric" instability end up being the same up to a rotation about an axis of symmetry. In the qualitative discussion that follows, however, we will focus on the Penrose conditions themselves and the nature of the self-energy at zero frequency.

\footnotetext{
${ }^{9}$ For example, a small sampling of references we have found useful is Refs. [25, 26, 31].
} 


\section{Qualitative Origin of Instabilities}

We will now give a brief qualitative review of the origin of the instabilities we have discussed, in part because we do not know of a really good qualitative review in the nonrelativistic plasma literature (especially of how the instability is saturated in the Abelian case), and in part to emphasize in a few cases differences for ultra-relativistic plasmas. In the context of the QCD plasma literature, a brief sketch is given by Mrówczyński in Ref. [4], which we will expand upon. Understanding how the instabilities are generated is useful for intuition in understanding what types of instabilities may arise for a given $f(\boldsymbol{p})$.

We will start with a formal observation about Condition 1 for Weibel instabilities. It is useful to integrate the formula $(2.8)$ by parts to obtain ${ }^{10}$

$$
\begin{array}{r}
\Pi^{i j}(\omega, \boldsymbol{q})=e^{2} \int_{\boldsymbol{p}} f(\boldsymbol{p})\left\{\frac{v}{p}\left[\delta^{i j}-\frac{q^{i} v^{j}+q^{j} v^{i}}{-\omega+\boldsymbol{v} \cdot \boldsymbol{q}-i \epsilon}+\frac{\left(-\omega^{2}+q^{2}\right) v^{i} v^{j}}{(-\omega+\boldsymbol{v} \cdot \boldsymbol{q}-i \epsilon)^{2}}\right]\right. \\
\left.+\frac{d v}{d p} \frac{\omega^{2} v^{i} v^{j}}{(-\omega+\boldsymbol{v} \cdot \boldsymbol{q}-i \epsilon)^{2}}\right\} .
\end{array}
$$

The last term vanishes for ultra-relativistic theories, for which $v=1$ and $d v / d p=0$. The interesting property of this expression is that, if one were to ignore the $i \epsilon$ prescription, the integrand above would be positive semi-definite for $\omega=0$. If not for the contribution near the singularity, $\Pi^{i j}(0, \hat{\boldsymbol{q}})$ could never have a negative eigenvalue. To see this, consider any unit polarization vector $\varepsilon$ and use (2.18) to write

$$
\varepsilon^{i} \Pi^{i j}(0, \boldsymbol{q}) \varepsilon^{j}=e^{2} \int_{\boldsymbol{p}} \frac{v}{p} f(\boldsymbol{p}) \frac{\left[\varepsilon^{2}(\boldsymbol{v} \cdot \boldsymbol{q})^{2}-2(\boldsymbol{\varepsilon} \cdot \boldsymbol{q})(\boldsymbol{\varepsilon} \cdot \boldsymbol{v})(\boldsymbol{v} \cdot \boldsymbol{q})+q^{2}(\varepsilon \cdot \boldsymbol{v})^{2}\right]}{(\boldsymbol{v} \cdot \boldsymbol{q}-i \epsilon)^{2}},
$$

whose integrand, if not for the $i \epsilon$ prescription, would always be non-negative by the vector inequality

$$
a^{2}(\boldsymbol{b} \cdot \boldsymbol{c})^{2}-2(\boldsymbol{a} \cdot \boldsymbol{b})(\boldsymbol{b} \cdot \boldsymbol{c})(\boldsymbol{c} \cdot \boldsymbol{a})+b^{2}(\boldsymbol{a} \cdot \boldsymbol{c})^{2} \geq 0 .
$$

The conclusion is that, in the perturbative limit, the origin of the Weibel instability must come completely from hard particles with $\boldsymbol{v}$ perpendicular to $\boldsymbol{q}$, which is when the $i \epsilon$ prescription plays a role. All other hard particles are stabilizing. As we shall review, the destabilizing particles with $\boldsymbol{v}$ perpendicular to $\boldsymbol{q}$ are just the perturbative limit of particles whose motions in the $\boldsymbol{q}$ direction are trapped by the fields. The importance of trapped particles to discussions of instabilities, and their relation to singularities of integrals in perturbative calculations, has a long history starting with the somewhat analogous case of electric instabilities in one dimension $[29,30]$. Note also that the effect of the stabilizing particles in (2.19) generically diverges as $\cos \theta \rightarrow 0$ like $d(\cos \theta) / \cos ^{2} \theta$, where $\theta$ is the angle between $\boldsymbol{p}$ and $\boldsymbol{q}$.

To understand all this qualitatively, consider first a gas of non-interacting hard particles in zero field, as shown in Fig. 3a. By our parity assumption, there is no current $\boldsymbol{j}(\boldsymbol{x})$ associated with these particles because, for all the particles going in one direction, there are

${ }^{10}$ Here and henceforth, we implicitly assume free hard particle dispersion relations are isotropic so that the direction of $\boldsymbol{v}$ is the same as that of $\boldsymbol{p}$. Kinetic theory is sometimes used to describe the effects of hard modes in classical thermal field theories on a discrete lattice, where this assumption would not be true. 


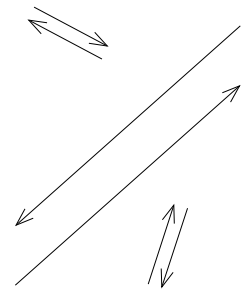

(a)

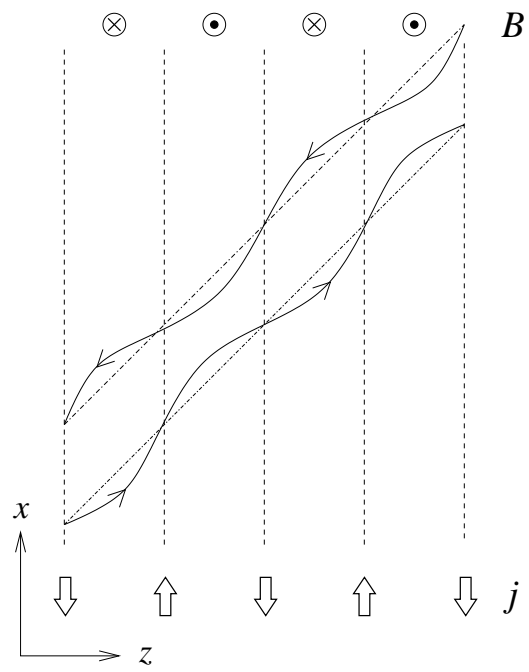

(b)

FIG. 3: (a) Parity opposites in a gas of free-streaming particles. (b) Small deviations from straightline trajectories of untrapped (stabilizing) particles in the background of a small magnetic field $\boldsymbol{B}=\mathcal{B} \mathbf{e}_{y} \sin (q z)$. The arrows show the direction of motion of positively charged particles, or the direction opposite to that of negatively charged particles. The direction $( \pm y)$ of the magnetic field is shown at the top, and the vertical dashed lines correspond to $\boldsymbol{B}(z)=0$. The direction $( \pm x)$ of the average current $\boldsymbol{j}(z)$ caused by the particles is shown at the bottom.

just as many particles going in the other direction. Now turn on a small magnetic field $\boldsymbol{B}$ with wave-vector $\boldsymbol{q}$, which we shall take to point in the $z$ direction. Take $\boldsymbol{B}$ to be in the $\pm y$ direction with $\boldsymbol{B}=\mathcal{B e}_{y} \sin (q z)$, as depicted in Fig. 3 b, where $\boldsymbol{e}_{y}$ is the unit vector in the $y$ direction. We'll take the vector field $\boldsymbol{A}$ to be in the $\pm x$ direction, with $\boldsymbol{A}=\mathcal{A e}_{x} \cos (q z)$ and $\boldsymbol{B}=\boldsymbol{\nabla} \times \boldsymbol{A}$. Magnetic forces from the small $\boldsymbol{B}$ field will make charged particles slightly wiggle around straight-line trajectories. The small wiggles in direction will cause the $x$ component $j_{x}$ of the current to be larger in some places and smaller in others, compared to the value obtained from the straight-line trajectory. This is shown at the bottom of the figure. These currents set up a magnetic field that opposes the original magnetic field and so stabilize against its growth.

The radius of curvature of particle trajectories in the plane perpendicular to the magnetic field is

$$
R=\frac{p_{\perp}}{e B},
$$

where $p_{\perp}$ is the momentum in that plane. It's easy to estimate ${ }^{11}$ that $\Delta v_{x} \sim e A v / p$ so that the effect described gives a contribution to $j_{x}$ of order $e^{2} A \int_{\boldsymbol{p}} f v / p$. This contribution to the current is finite in the $\cos \theta \rightarrow 0$ limit and does not explain the small $\cos \theta$ divergence discussed earlier.

Besides this variation in the magnitude of $j_{x}$ for each particle at a given position, there

11 The field has roughly the same order of magnitude over a range of $z$ close to half a period $\lambda / 2=\pi / q$ of the magnetic field. Simple geometry gives that the resulting small change in $v_{x}$ over half a period is then of order $v \lambda / R$, which in turn is $\sim e v B / p q \sim e v A / p$. 


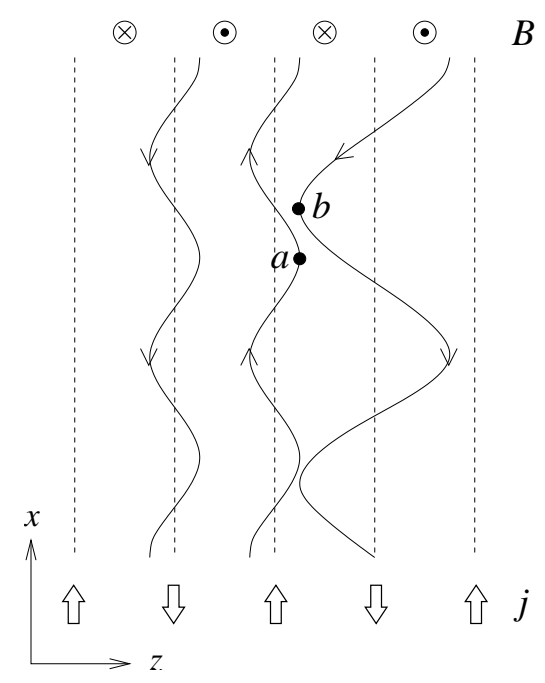

FIG. 4: The same situation as in Fig. $3 \mathrm{~b}$ but for particles with small enough $v_{z}$ that their $z$ motion is trapped.

is a second effect: the amount of time the particle spends at a given $z$ coordinate changes, because the component $v_{z}$ of its velocity also wiggles. The particles will spend more of their time in regions of $z$ where $v_{z}$ is smaller (which are the regions where $v_{x}$ is bigger). The slowing/speeding effect of the $z$ motion therefore generates a contribution to the average current $\boldsymbol{j}(z)$ which is also in the directions shown in Fig. 3b. One may parametrically estimate that the variation $\Delta v_{z}$ of $v_{z}$ has relative size of $\operatorname{order}^{12}\left(\Delta v_{z}\right) / v_{z} \sim e A v v_{x} /\left(p v_{z}^{2}\right)$, giving

$$
j^{x} \sim e^{2} A \int_{p} f \frac{v v_{x}^{2}}{p v_{z}^{2}}
$$

This gives the $d(\cos \theta) / \cos ^{2} \theta$ divergence, since $v_{z}=v \cos \theta$ in our choice of coordinates. This effect is also stabilizing.

So far we have focused on the contribution from particles that stabilize magnetic fluctuations. Now consider particles whose initial velocities are very close to being orthogonal to $\boldsymbol{q}$. The curvature of trajectories caused by the magnetic field will trap the $z$ motion of such particles, as depicted in Fig. 4. The two trajectories shown on the left of the figure generate currents opposite those shown in Fig. 3, which therefore generate a magnetic field that adds to the existing one and contributes to instability. The contribution of the trajectory on the far right can be appreciated as follows. Imagine initially two particles, one at the point marked $a$ and one at the point marked $b$, have parity-opposite momentum in the $\pm x$ directions. Then imagine suddenly turning on the magnetic forces in the problem. The upward moving particle at $a$ will follow the middle trajectory and continue to give an upward contribution to $\boldsymbol{j}$ in roughly the same region of $z$. The downward particle at $b$, whose contribution to $\boldsymbol{j}$ was originally canceling, will drift far away and so spend only a portion of its time close to its original $z$. That leads to the generation of a new upward average current in the region of their original $z$.

${ }^{12}$ Motion in a magnetic field conserves $|\boldsymbol{v}|$. This means that $v_{x} \Delta v_{x}+v_{z} \Delta v_{z} \simeq 0$, so that $\left(\Delta v_{z}\right) / v_{z} \sim$ $\left(\Delta v_{x}\right) v_{x} / v_{z}^{2}$. Then, use the previous estimate of $\Delta v_{x}$. 


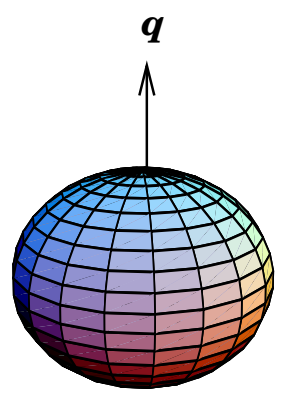

(a)

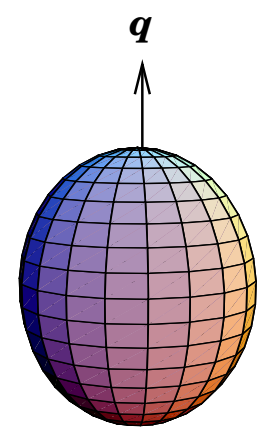

(b)

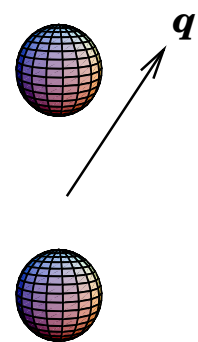

(c)

FIG. 5: Some qualitatively different examples of anisotropic distributions $f(\boldsymbol{p})$ together with some specific examples of directions for $\boldsymbol{q}$. The plots show the shape of surfaces of constant $f$ in $\boldsymbol{p}$ space.

For very small magnetic fields, only a perturbatively small fraction of phase space, corresponding to very small $\left|v_{z}\right|$, is trapped. However, each such particle contributes to the current separation an $O(e)$ amount, unsuppressed by the size of $A$. In contrast, the untrapped particles, making up almost all of phase space, each contribute a stabilizing influence; but the size of that stabilizing influence is perturbatively small (that is, suppressed by a power of the size of $A$ ). It is for this reason that trapped and untrapped contributions can be the same order of magnitude even in the perturbative limit. ${ }^{13}$

In the case of isotropic hard distributions $f(\boldsymbol{p})$, the contributions of trapped (destabilizing) and untrapped (stabilizing) particles must cancel to give the isotropic result $\Pi^{i j}(0, \hat{\boldsymbol{q}})=0$. This delicate cancellation allows us to understand the presence or absence of magnetic instabilities in certain simple situations. If we start with an isotropic distribution and, for a given $\boldsymbol{q}$, remove some of the untrapped particles, then we must have magnetic instability. This is the case for the $\boldsymbol{q}$ shown for an oblate distribution in Fig. 5a. If we instead add untrapped particles, we must have stability, such as for the $\boldsymbol{q}$ shown for a prolate distribution in Fig. 5b. In 5c, assume that the number of particles outside the surfaces is insignificant. Then this is another example where there is no magnetic instability (for the $\boldsymbol{q}$ shown) because the number of trapped particles is insignificant.

Finally, we will review the related picture for electric instabilities. We will focus on $\Pi^{00}(0, \hat{\boldsymbol{q}})$, which gives the charge response to a sinusoidal electric potential. Consider a small static electric potential $A^{0}=\phi \cos (q z)$ and the corresponding electric field $\boldsymbol{E}=-\boldsymbol{\nabla} A^{0}=$ $\mathcal{E} \boldsymbol{e}_{z} \sin (q z)$. First think about the one dimensional problem - that is, particles moving in the

${ }^{13}$ Details depend on the distribution $f(\boldsymbol{p})$ and the direction $\hat{\boldsymbol{q}}$. However, for generic distributions and generic directions $\hat{\boldsymbol{q}}$, one can parametrically estimate that trapping typically occurs in weak fields when $R|\cos \theta| \lesssim 1$. From $(2.21)$, that is $|\cos \theta| \lesssim \sqrt{e A / p}$, which indeed is formally arbitrarily small in the perturbative limit $e \rightarrow 0$. In a hand-waving sense, the $i \epsilon$ prescription in the denominator of (2.19) can then be thought of as being of order $\sqrt{e A / p}$. In any case, one can use (2.22) and integrate down to this $|\cos \theta|$ to parametrically estimate the stabilizing untrapped contribution to be of order $e \int_{p} f v / \sqrt{e A / p}$. One finds a similar estimate for the order of magnitude of the trapped contribution, and the leading $O\left(e^{1 / 2}\right)$ pieces of these contributions cancel to leave the perturbative $O\left(e^{2}\right)$ result for $\Pi^{i j}(0, \boldsymbol{q})$. 


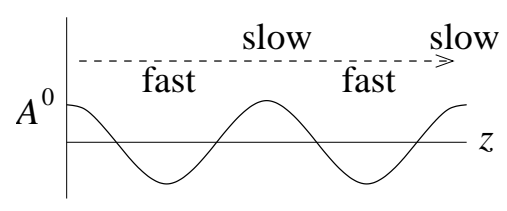

(a)

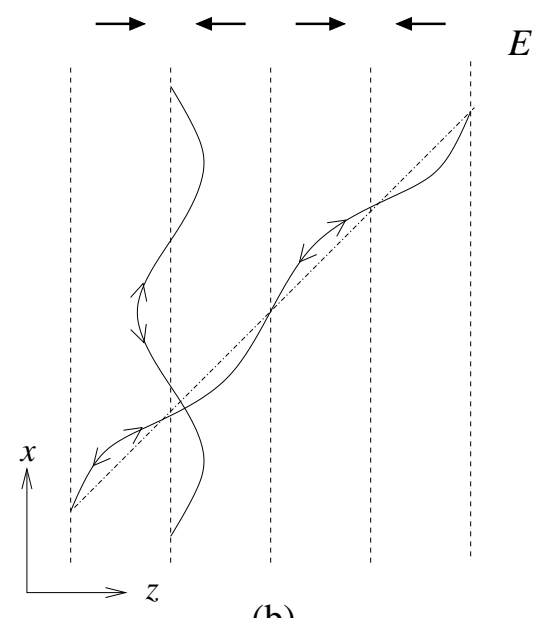

(b)

FIG. 6: Qualitative picture of particle motion in a sinusoidal electric potential. (a) An untrapped non-relativistic particle in one dimension. (b) Untrapped and trapped particles in two or three dimensions.

$z$ direction, depicted in Fig. 6. If the field is small, typical particles will be able to move over the electric potential barriers. By energy conservation, however, positively charged particles will move slower at the maxima of $A^{0}$ and faster at the minima. So they will spend more of their time near the maxima, and so their contribution to the average charge density will be greatest there, which will generate a yet larger $A^{0}$ there. (Negatively charged particles will spend more time at the minima of $A^{0}$, which will also enhance the magnitude of $A^{0}$.) Untrapped particles are therefore destabilizing, which is the opposite of what happened in the magnetic case. In contrast, positively charged particles which have tiny velocities are trapped in the minima of $A^{0}$ and so contribute positively to the charge density there, which reduces $A^{0}$. The electrically trapped particles are stabilizing. The one-dimensional situation is often discussed in the non-relativistic case but is not relevant to ultra-relativistic particles because such particles always move with $v=1$. However, in the three-dimensional case, $v_{z}$ can become smaller or larger as particles move through minima and maxima even while $v$ remains the same, just as in our discussion of the magnetic case. Examples of electrically trapped and untrapped particles are given in Fig. 6b.

Isotropic distributions are electrically stable and associated with a positive value of $-\Pi^{00}(0, \boldsymbol{q})$ given by the squared Debye mass $m_{\mathrm{D}}^{2}$. Small deviations from isotropy will not satisfy Condition 2 of Sec. II B, but significant deviations from isotropy can.

An example where one can simply conclude that Condition 2 for electric instability is not satisfied is an oblate distribution, such as shown in Fig. 5a, with $\hat{\boldsymbol{q}}$ pointing along the axis of axial symmetry, which we will take to be the $z$ axis. For reasons similar to those given previously for the magnetic case, except that now the role of trapped and untrapped particles is reversed, $-\Pi^{00}\left(0, \boldsymbol{e}_{z}\right)>0$. Axial symmetry and the Ward identity (2.9) imply $\Pi^{0 i}\left(0, \boldsymbol{e}_{z}\right)$ vanishes, and so Condition 2 will not be satisfied in this situation. 


\section{Saturation of Instabilities}

When discussing the linear response of the gauge fields, there is no significant difference between Abelian and non-Abelian theories. When the instabilities grow large enough to become non-linear, there is. It is useful to understand qualitatively how instabilities saturate in the Abelian case, in order to contrast it with the non-Abelian case.

The saturation of Abelian electric instabilities is straightforward to understand qualitatively. Return to Fig. 6 and imagine making the electric field arbitrarily large (holding $q$ fixed). Then essentially all particles will become trapped in the $\boldsymbol{q}$ direction near the bottom of the potential wells, which means there will be no instability. So, if there was originally an instability, the field will eventually grow large enough for it to saturate. This will happen very roughly when the electric potential energy $e A^{0}$ becomes of order the typical energy associated with the motion of the particles in the $\boldsymbol{q}$ direction. In the non-relativistic limit, that is

$$
A^{0} \sim \frac{p_{\|}^{2}}{e M}, \quad E \sim \frac{p_{\|}^{2} q}{e M},
$$

where $M$ is the particle mass and $p_{\|}$indicates the typical value of the component of $p$ parallel to $\boldsymbol{q}$. In the ultra-relativistic limit,

$$
A^{0} \sim \frac{p_{\|}^{2}}{e p}, \quad E \sim \frac{p_{\|}^{2} q}{e p} .
$$

This saturation was used long ago to construct non-linear wave solutions in the nonrelativistic Abelian case, known as Bernstein-Greene-Kruskal waves [30].

To qualitatively understand the saturation of magnetic instabilities, return to the pictures of Figs. 3 and 4, but now make the magnetic fields arbitrarily large (holding $\boldsymbol{q}$ fixed). In this case, the typical radius of curvature (2.21) will be small compared to the distance in $z$ over which $\boldsymbol{B}(z)$ changes sign. Instead of looking like Fig. 3b or 4, typical trajectories will instead look like Fig. 7. The particles follow small, nearly circular orbits in the magnetic field which slowly drift in the direction indicated due to slightly higher curvature on the side where $\boldsymbol{B}$ is slightly larger. This sets up currents, shown at the bottom of the figure, which create magnetic fields that oppose the original field. Essentially all particles are therefore stabilizing in the high field limit. So, once again, if there was originally an instability, the fields will eventually grow large enough for it to saturate. That will happen very roughly when the radius of curvature (2.21) becomes of order the wavelength $\lambda \sim 1 / q$ of the field, 


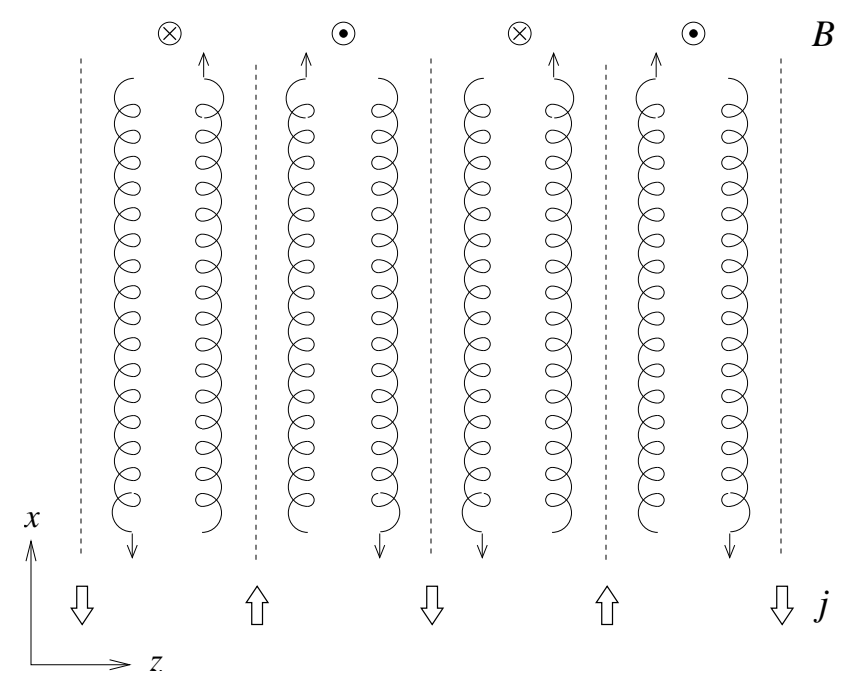

FIG. 7: Trajectories in an arbitrarily large magnetic field. The size of the orbits and the rate of their drift has been exaggerated.

$\mathrm{SO}^{14}$

$$
B \sim \frac{p_{\top} q}{e}, \quad A \sim \frac{p_{\top}}{e} .
$$

A simple mnemonic for cases where the hard particles can be described as excitations in a quantum field theory is to realize that, when acting on hard excitations of momentum $p$, the gauge term in the covariant derivative $D=\partial-i e A$ cannot be treated as a small perturbation to the derivative term when (roughly speaking) $A \gtrsim p / e$.

In contrast, if different unstable soft field modes with momenta of order $q$ can interact with each other, then linearity breaks down when

$$
A \sim \frac{q}{g}
$$

instead of $A \sim p / g$, where we have switched notation from $e$ to $g$ as a way of emphasizing that this is special to the non-Abelian case. We will see a specific example in Sec. IV.

14 This and (2.23) represent estimates in generic cases of significant anisotropy. If, for example, the anisotropy is very tiny or $q$ is very close to its maximum unstable value, then a very tiny higher-order correction to the perturbative calculation might change instability to stability. A brief and somewhat different discussion of rough estimates of the saturation condition in the non-relativistic case for both magnetic and electric instabilities may be found in Ref. [33], as well as references therein. There the saturation condition is estimated in terms of time rather than distance scales by equating the "bounce" time for an oscillation of a trapped particle to the linearized growth rate of the instability. For generic situations and generic unstable $\boldsymbol{q}$, this is roughly equivalent to our rough conditions (2.23) and (2.24) if one assumes that the growth rate $\gamma$ is of order $v q$ (where we will no longer try to distinguish between $v_{\|}$and $v_{\perp}$ ). See, for example, Eqs. (1), with the condition $\omega_{\mathrm{B}} \sim \gamma$, and (114), with $k$ small, of Ref. [33]. The estimate $\gamma \sim v q$ (which is $\ll q$ in the non-relativistic limit) can be roughly understood by thinking of increasing $\omega=i \gamma$ from zero and noting that it first has an effect in the self-energy when $\omega \sim v q$, so that it significantly affects the integration over the denominators in (2.18). 
Even for the Abelian case, the picture of Fig. 7 is a bit simplistic. It assumes that only one unstable mode with a given $\boldsymbol{q}$ has been excited. In most situations, an entire spectrum of unstable modes will grow together and then interact with each other once they become non-perturbatively large according to (2.24). The idealized case of a single Abelian mode has been studied in the non-relativistic literature and certain non-linear wave solutions have been found, known as magnetic Bernstein-Greene-Kruskal (BGK) waves [32, 33]. The generation of these non-linear waves has also been investigated numerically [33-35], in some cases with only a single unstable mode significantly excited and in other cases with many.

\section{INSTABILITY OF THE PLANAR MOMENTUM DISTRIBUTION}

We will now focus in detail on the planar momentum distribution of (1.1):

$$
f(\boldsymbol{p}, \boldsymbol{x})=F\left(p_{\perp}\right) \delta\left(p_{z}\right) .
$$

\section{A. Magnetic instabilities}

1. $\boldsymbol{\omega}=\mathbf{0}$ magnetic stability analysis

To check for evidence of magnetic instabilities, first consider the self-energy (2.18) at $\omega=0$,

$$
\Pi^{i j}(0, \hat{\boldsymbol{q}})=e^{2} \int_{\boldsymbol{p}} f(\boldsymbol{p}) \frac{v}{p}\left[\delta^{i j}-\frac{\hat{q}^{i} \hat{p}^{j}+\hat{q}^{j} \hat{p}^{i}}{\hat{\boldsymbol{p}} \cdot \hat{\boldsymbol{q}}-i \epsilon}+\frac{\hat{p}^{i} \hat{p}^{j}}{(\hat{\boldsymbol{p}} \cdot \hat{\boldsymbol{q}}-i \epsilon)^{2}}\right] .
$$

The planar distribution $f(\boldsymbol{p})$ has support only in the $p_{x} p_{y}$ plane and is independent of the direction of $\boldsymbol{p}$ in that plane. We may therefore factor out the angular dependence of the integral and write

$$
\Pi^{i j}(0, \boldsymbol{q})=m_{\infty}^{2}\left\langle\delta^{i j}-\frac{\hat{q}^{i} \hat{p}^{j}+\hat{q}^{j} \hat{p}^{i}}{\hat{\boldsymbol{p}} \cdot \hat{\boldsymbol{q}}-i \epsilon}+\frac{\hat{p}^{i} \hat{p}^{j}}{(\hat{\boldsymbol{p}} \cdot \hat{\boldsymbol{q}}-i \epsilon)^{2}}\right\rangle_{\hat{\boldsymbol{p}} \in \text { plane }} .
$$

Because of the axi-symmetry of the problem, we can assume without loss of generality that $\boldsymbol{q}$ points in the $x z$ plane. Then $\boldsymbol{e}_{y}$ is an eigenvector of $\Pi^{i j}$ because of $y \rightarrow-y$ reflection symmetry. For $\omega=0$, the direction $\hat{\boldsymbol{q}}$ is also an eigenvector of $\Pi^{i j}$, corresponding to zero eigenvalue. Let $\hat{\boldsymbol{n}}$ be the orthogonal direction in the $x z$ plane, as depicted in Fig. 8. Let $\theta$ be the angle $\hat{\boldsymbol{q}}$ makes with the $z$ axis and $\phi$ the angle $\hat{\boldsymbol{p}}$ makes with the $x$ axis. Then, for $\sin \theta \neq 0$, the eigenvalues of $\Pi^{i j}(0, \hat{\boldsymbol{q}})$ are easily computed from (3.3) to be

$$
\begin{aligned}
\Pi^{\hat{\boldsymbol{q}} \hat{\boldsymbol{q}}}(0, \hat{\boldsymbol{q}}) & =0, \\
\Pi^{\hat{\boldsymbol{n}} \hat{\boldsymbol{n}}}(0, \hat{\boldsymbol{q}}) & =m_{\infty}^{2} \int_{0}^{2 \pi} \frac{d \phi}{2 \pi}\left[1+0+\frac{\cos ^{2} \theta}{\sin ^{2} \theta}\right]=\frac{m_{\infty}^{2}}{\sin ^{2} \theta} \\
\Pi^{y y}(0, \hat{\boldsymbol{q}}) & =m_{\infty}^{2} \int_{0}^{2 \pi} \frac{d \phi}{2 \pi}\left[1+0+\frac{\sin ^{2} \phi}{\sin ^{2} \theta(\cos \phi-i \epsilon)^{2}}\right]=m_{\infty}^{2}\left(1-\frac{1}{\sin ^{2} \theta}\right)=-m_{\infty}^{2} \cot ^{2} \theta .
\end{aligned}
$$




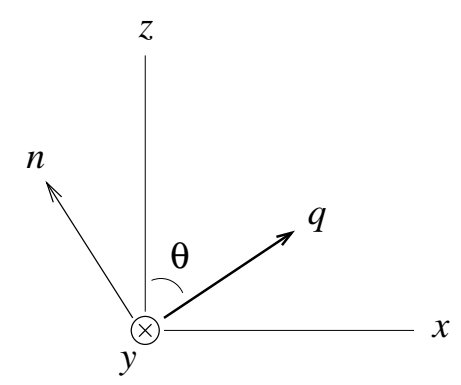

FIG. 8: Convention for labeling directions with respect to $\hat{\boldsymbol{q}}$, which is taken to lie in the $x z$ plane.

Only $\Pi^{y y}(0, \hat{\boldsymbol{q}})$ is negative. ${ }^{15}$ By Condition 1 of Sec. II B, there is a magnetic instability associated with $\boldsymbol{A}$ polarized in the $y$ direction (and so $\boldsymbol{B}$ in the $\hat{\boldsymbol{n}}$ direction) whenever

$$
q<q_{\max }(\theta) \equiv m_{\infty} \cot \theta
$$

It is easy to understand qualitatively why there is no magnetic instability associated with the $\boldsymbol{n}$ polarization of $\boldsymbol{A}$, which would correspond to the magnetic field $\boldsymbol{B}$ in the $\pm y$ direction. Magnetic instability is caused by trapped particles, which perturbatively are those with $\boldsymbol{p}$ orthogonal to $\boldsymbol{q}$. For the planar distribution with $\boldsymbol{q}$ in the $x z$ plane, the only trapped particles are those with $\boldsymbol{p}$ pointing in the $\pm y$ direction (for $\sin \theta \neq 0$ ). If $\boldsymbol{B}$ is also in the $\pm y$ direction, then the motion of these particles is not affected by the magnetic field, and so there is no destabilizing contribution.

\section{Magnetic instability growth rate in the ultra-relativistic limit}

We can get simple expressions for $\Pi^{i j}(\omega, \boldsymbol{q})$ for general $\omega$ if we specialize to the ultrarelativistic limit, which we will now do. In this case, it is straightforward to keep the frequency dependence in the manipulations that led to from (2.18) to (3.3) for the selfenergy, giving

$$
\Pi^{i j}(\omega, \boldsymbol{q})=m_{\infty}^{2}\left\langle\delta^{i j}-\frac{\hat{q}^{i} \hat{p}^{j}+\hat{q}^{j} \hat{p}^{i}}{-\eta+\hat{\boldsymbol{p}} \cdot \hat{\boldsymbol{q}}-i \epsilon}+\frac{\left(-\eta^{2}+1\right) \hat{p}^{i} \hat{p}^{j}}{(-\eta+\hat{\boldsymbol{p}} \cdot \hat{\boldsymbol{q}}-i \epsilon)^{2}}\right\rangle_{\hat{\boldsymbol{p}} \in \text { plane }}
$$

where

$$
\eta \equiv \frac{\omega}{q}
$$

Since there is no suggestion of magnetic instabilities for other polarizations for $\sin \theta \neq 0$, let us focus on $\Pi^{y y}$. The nice thing about the simple planar distribution is that one can find a simple closed form solution from (3.6). Performing the $\phi$ integrals, one finds

$$
\Pi^{y y}(\omega, \boldsymbol{q})=m_{\infty}^{2}\left\{1+\frac{1-\eta^{2}}{\sin ^{2} \theta}\left[\left(1-\frac{\sin ^{2} \theta}{\eta^{2}}\right)^{-1 / 2}-1\right]\right\} .
$$

\footnotetext{
15 This instability corresponds to poles in $\Delta_{A}$ of Ref. [17] in the $\xi \rightarrow+\infty$ limit. The possibility of instability in this mode was overlooked in Ref. [6].
} 


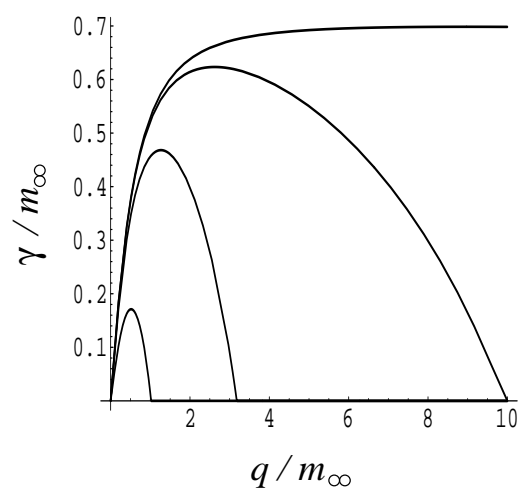

(a)

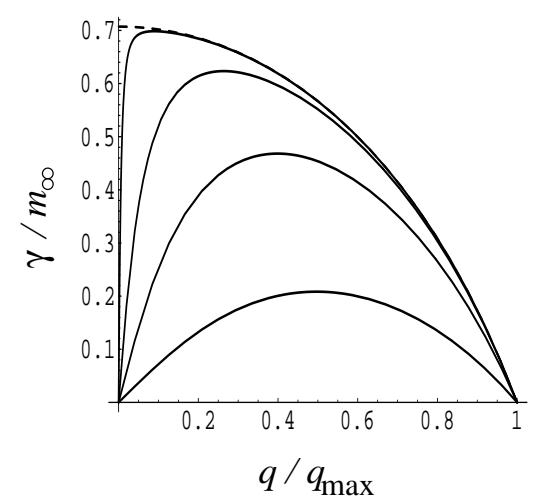

(b)

FIG. 9: The growth rate vs. $q$ for magnetic instabilities for the planar distribution for various values of $\theta$. From the top down, the values are $\sin \theta=0.01,0.1,0.3,0.65$. The small dashed line at the top of (b) shows the $\sin \theta \rightarrow 0$ limit.

The corresponding dispersion relation is

$$
\left(-\eta^{2}+1\right) q^{2}+\Pi^{y y}(\omega, \boldsymbol{q})=0 .
$$

By re-arranging terms algebraically and squaring, one can convert this into a cubic equation in $\eta^{2}$, a subset of whose roots are the solutions to (3.8b). However, the explicit closed form of that solution is no more enlightening than a numerical solution. In Fig. 9a, we show the result for the growth rate $\gamma=\operatorname{Im} \omega=q \operatorname{Im} \eta$ of the unstable solution as a function of $q$ for various values of $\theta$. It is clear that the largest growth rates correspond to $\sin \theta \rightarrow 0$, which is not surprising since, for $\theta=0$, all particles in the planar distribution become trapped particles, contributing to destabilization. Plotting $\gamma / m_{\infty}$ vs. $q / q_{\max }$ in Fig. $9 \mathrm{~b}$, with $q_{\max }$ taken from (3.5), it is clear that there is a limiting behavior as $\sin \theta \rightarrow 0$. For $\operatorname{small} \sin \theta$, the dispersion relation $(3.8 \mathrm{~b})$ can be more compactly solved to give

$$
\frac{\gamma}{m_{\infty}} \simeq f_{1}\left(\frac{q}{m_{\infty}}\right)+f_{2}\left(\frac{q}{q_{\max }}\right)-\frac{1}{\sqrt{2}},
$$

where

$$
\begin{aligned}
& f_{1}(z)=\frac{1}{2}\left\{\left[\left(1+2 z^{2}\right)^{2}+8 z^{2}\right]^{1 / 2}-\left(1+2 z^{2}\right)\right\}^{1 / 2}, \\
& f_{2}(z)=\frac{1-z^{2}}{\left(2-z^{2}\right)^{1 / 2}} .
\end{aligned}
$$

Note that $m_{\infty} \simeq q_{\max } \sin \theta$ for small $\sin \theta . f_{1}(z)$ runs from 0 to $1 / \sqrt{2}$ and $f_{2}(z)$ from $1 / \sqrt{2}$ to 0 as $z$ runs from 0 to $\infty$. For $q \ll m_{\infty}$, one gets $\gamma \simeq q$ in this small $\sin \theta$ limit. The maximum growth rate for a given small $\sin \theta$ occurs at $q \simeq m_{\infty}^{2}\left(\frac{2}{3} \sin ^{2} \theta\right)^{1 / 4}$.

For $\sin \theta=0$, the result becomes simply

$$
\frac{\gamma}{m_{\infty}}=f_{1}\left(\frac{q}{m_{\infty}}\right) \quad(\sin \theta=0)
$$

which, as $q \rightarrow \infty$, approaches the maximum growth rate for the planar problem of $\gamma=$ $m_{\infty} / \sqrt{2}$. By symmetry the behavior of $\Pi^{x x}$ must be the same as that of $\Pi^{y y}$ at $\sin \theta=0$, and so there are two rather than one magnetically unstable modes at exactly $\sin \theta=0$. 


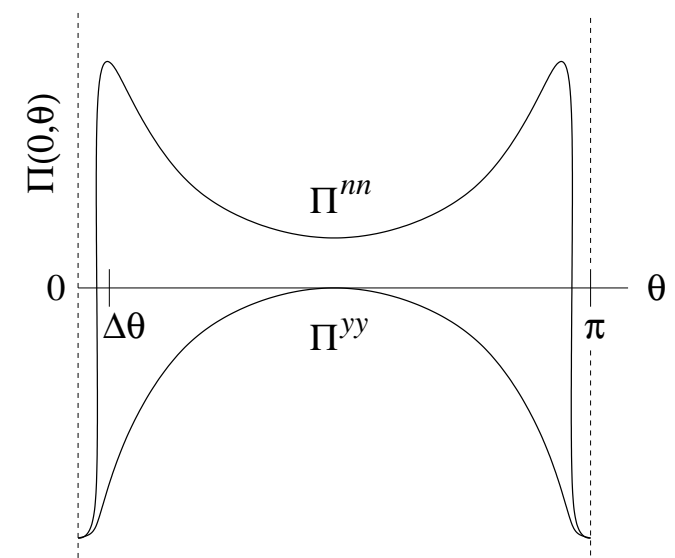

FIG. 10: Qualitative behavior of $\Pi^{\hat{\boldsymbol{n}} \hat{\boldsymbol{n}}}(0, \hat{\boldsymbol{q}})$ and $\Pi^{y y}(0, \hat{\boldsymbol{q}})$ vs. $\hat{\boldsymbol{q}}$ for a planar distribution with small thickness $\Delta \theta$. The curves for $\sin \theta \gg \Delta \theta$ depict the formulas of (3.4).

\section{Thick planar distributions and related matters}

One can now ask what happens if the planar distribution has a tiny thickness, so that $\delta\left(p_{z}\right)$ is replaced by something with a small but finite width $\Delta p_{z}$. Let $\Delta \theta \sim\left(\Delta p_{z}\right) / p$ be the angular width of the distribution, where $p$ is the momentum scale that dominates $f(\boldsymbol{p})$, and assume $\Delta \theta \ll 1$. The effect this will have is to smear out the $\theta$ dependence of previous results over $\Delta \theta$. In particular, $\Pi^{y y}(0, \hat{\boldsymbol{q}})$ will now be finite for $\hat{\boldsymbol{q}}$ in the $\pm z$ direction, with (3.5) replaced at $\theta=0$ by

$$
q_{\max }(\theta=0) \sim \frac{m_{\infty}}{\Delta \theta} .
$$

Also, there will now be two unstable modes for $\sin \theta \lesssim \Delta \theta$ rather than just at $\sin \theta=0$. The qualitative behavior of $\Pi^{x x}(0, \hat{\boldsymbol{q}})$ and $\Pi^{y y}(0, \hat{\boldsymbol{q}})$ as a function of $\theta$ is shown in Fig. 10.

Though the preceding is a correct analysis of "magnetic" instabilities as defined by Condition 1 of Sec. IIB, it is qualitatively a bit misleading. Instead of focusing on the zero frequency self-energy used in Condition 1, let us make some very rough qualitative arguments about the self-energy at the non-zero frequency $\omega=i \gamma$ corresponding to the growing unstable mode. The origin of the formal divergence of the result $(3.4 \mathrm{c})$ for $\Pi^{y y}(0, \boldsymbol{q})$ as $\theta \rightarrow 0$ is in the denominators $(-\eta+\hat{\boldsymbol{p}} \cdot \hat{\boldsymbol{q}}-i \epsilon)^{2}$ for the zero-thickness $(\Delta \theta=0)$ planar distribution. For $\eta=0$, this is negative (destabilizing) only when $\hat{\boldsymbol{p}} \cdot \boldsymbol{q}=0$. For $\eta=i \gamma / q$, it is instead destabilizing when $\hat{\boldsymbol{p}} \cdot \boldsymbol{q} \lesssim \gamma / q$. For $\boldsymbol{q}$ close to the $z$ axis, the average value of $(\hat{\boldsymbol{p}} \cdot \hat{\boldsymbol{q}})^{2}$ is of order $\theta^{2}$. The qualitative effect of non-zero $\gamma$ is therefore similar to thickening out the planar distribution by $\Delta \theta \sim \gamma / q$. For small $\theta$, we have found magnetic instabilities with $\gamma / q \sim 1$ when $q \ll q_{\max }$, which is therefore similar to $\Delta \theta \sim 1$. We therefore expect that for small $\theta \ll 1$ and $q \ll q_{\max }$, the smearing due to non-zero $\eta$ should be enough to allow an $x$-polarized unstable solution that looks just like the $y$-polarized solution we have already found. Indeed, we shall discover just such a solution in the next section, where we turn to the "electric" instabilities associated with Condition 2 of Sec. II B. 


\section{B. "Electric" instabilities in the ultra-relativistic limit}

\section{1. $\boldsymbol{\omega}=\mathbf{0}$ electric stability analysis}

Let us look for electric instabilities by checking Condition 2 of Sec. II B. First consider $\Pi^{00}(0, \boldsymbol{q})$. Starting from the general expression (2.8) for the self-energy and integrating by parts, one finds

$$
\Pi^{00}(0, \hat{\boldsymbol{q}})=e^{2} \int_{\boldsymbol{p}} f(\boldsymbol{p})\left[\frac{1}{v p}\left(-1+\frac{1}{(\hat{\boldsymbol{p}} \cdot \hat{\boldsymbol{q}}-i \epsilon)^{2}}\right)+\frac{1}{v^{2}} \frac{d v}{d p}\right] .
$$

Let's focus on the case $\sin \theta \neq 0$. For the planar distribution function, the angular integral of $(\hat{\boldsymbol{p}} \cdot \hat{\boldsymbol{q}}-i \epsilon)^{-2}$ over $\hat{\boldsymbol{p}}$ in the $x y$ plane gives zero, and one finds the $\hat{\boldsymbol{q}}$-independent result

$$
\Pi^{00}(0, \hat{\boldsymbol{q}})=e^{2} \int_{\boldsymbol{p}} f(\boldsymbol{p})\left[-\frac{1}{v p}+\frac{1}{v^{2}} \frac{d v}{d p}\right] .
$$

This result gives zero in the non-relativistic limit. We will focus on the ultra-relativistic limit, where

$$
\Pi^{00}(0, \hat{\boldsymbol{q}})=-m_{\infty}^{2}
$$

which is negative and so stabilizing.

But now we need to examine $\Pi^{0 i}(0, \hat{\boldsymbol{q}})$. From $y \rightarrow-y$ reflection symmetry, one has $\Pi^{0 y}(0, \hat{\boldsymbol{q}})=0$ and from the Ward identity, $\Pi^{0 \hat{\boldsymbol{q}}}(0, \hat{\boldsymbol{q}})=0$. It remains to calculate $\Pi^{0 \hat{\boldsymbol{n}}}(0, \hat{\boldsymbol{q}})$. One can use (2.16), but we find it simpler to proceed similarly to earlier calculations with the planar distribution. Integrating (2.8) for the self-energy by parts,

$$
\Pi^{0 i}(0, \hat{\boldsymbol{q}})=e^{2} \int_{\boldsymbol{p}} \frac{f(\boldsymbol{p})}{p} \frac{\hat{v}^{i}-\hat{q}^{i} \hat{\boldsymbol{v}} \cdot \hat{\boldsymbol{q}}}{(\hat{\boldsymbol{v}} \cdot \hat{\boldsymbol{q}}-i \epsilon)^{2}} .
$$

In particular, we can write

$$
\Pi^{0 \hat{\boldsymbol{n}}}(0, \hat{\boldsymbol{q}})=e^{2} \int_{\boldsymbol{p}} \frac{f(\boldsymbol{p})}{p}\left\langle\frac{(-\cos \theta \cos \phi)}{(\sin \theta \cos \phi-i \epsilon)^{2}}\right\rangle_{\phi}=-\frac{i \cos \theta}{\sin ^{2} \theta} e^{2} \int_{\boldsymbol{p}} \frac{f(\boldsymbol{p})}{p} .
$$

In the ultra-relativistic limit, this is just

$$
\Pi^{0 \hat{\boldsymbol{n}}}(0, \hat{\boldsymbol{q}})=-i m_{\infty}^{2} \frac{\cos \theta}{\sin ^{2} \theta} .
$$

Now we can apply Condition 2 of Sec. II B, which indicates an electric instability if

$$
q^{2}-\Pi^{00}(0, \hat{\boldsymbol{q}})+\Pi^{0 i}(0, \hat{\boldsymbol{q}})\left[q^{2}+\Pi(0, \boldsymbol{q})\right]_{i j}^{-1} \Pi^{j 0}(0, \hat{\boldsymbol{q}})<0
$$

Making use of the result $(3.4 \mathrm{~b})$ for $\Pi^{\hat{\boldsymbol{n}} \hat{\boldsymbol{n}}}(0, \hat{\boldsymbol{q}})$, this condition becomes, for the ultra-relativistic planar distribution,

$$
q^{2}+m_{\infty}^{2}-\frac{m_{\infty}^{4} \cot ^{2} \theta}{q^{2} \sin ^{2} \theta+m_{\infty}^{2}}<0
$$


The left-hand side is minimized for $q=0$, in which case the inequality gives $1-\cot ^{2} \theta<0$. So, there will be some mode of electric instability whenever $\hat{\boldsymbol{q}}$ lies within 45 degrees of the $z$ axis. ${ }^{16}$ For a given $\theta$, the maximum $q$ which gives an instability is given by (3.21) as

$$
q_{\max }=m_{\infty}\left[\frac{\cos \theta\left(4+\cos ^{2} \theta\right)^{1 / 2}-\sin ^{2} \theta-1}{2 \sin ^{2} \theta}\right]^{1 / 2} .
$$

Since $-\Pi^{00}(0, \hat{\boldsymbol{q}})>0$, which is stabilizing, the "electric" instability in this case is not due to the simple picture of electric instabilities discussed in Sec. II C but instead depends on the coupling of charge and current fluctuations through $\Pi^{i 0}$. An example of a distribution with an electric instability whose origin does have the simple interpretation of Sec. II C [having $\left.\Pi^{i 0}(0, \hat{\boldsymbol{q}})=0\right]$ is given in Appendix C.

\section{Electric instability growth rate}

In Appendix D, we briefly summarize the calculation of the dispersion relation for polarizations of $\boldsymbol{A}$ in the $x z$ plane ( $\hat{\boldsymbol{q}} \hat{\boldsymbol{n}}$ plane), just as (3.8) gave the dispersion relation for the $y$ polarization. The result is ${ }^{17}$

$$
\begin{aligned}
\cos ^{2} \theta\left(1-\frac{\sin ^{2} \theta}{\eta^{2}}\right)^{-1} & {\left[\left(1-\frac{\sin ^{2} \theta}{\eta^{2}}\right)^{-1 / 2}-1\right] } \\
+ & \eta^{2}\left[1+\left(1-\eta^{2}\right) Q^{2}\right]\left[\left(1-\frac{\sin ^{2} \theta}{\eta^{2}}\right)^{-1 / 2}-1-Q^{2} \sin ^{2} \theta\right]=0
\end{aligned}
$$

where we have introduced the dimensionless variable $Q \equiv q / m_{\infty}$. The unstable solutions are plotted in Fig. 11 for various choices of $\theta$. Unlike the magnetic case, there are no solutions for angles more than $45^{\circ}$ from the $z$ axis. For any given $\boldsymbol{q}$, the maximum instability occurs for $\sin \theta \rightarrow 0$, which is a limit in which the unstable solution to (3.23) becomes

$$
\frac{\gamma}{m_{\infty}} \rightarrow f_{1}\left(\frac{q}{m_{\infty}}\right)
$$

which is just the same as (3.12) for the magnetic case. One may check (see Appendix D) that the corresponding polarization of $\boldsymbol{E}$ is in the $x$ direction. This is just the solution previously predicted for small $\sin \theta$ in Sec. III A 3.

One may also explore taking $\sin \theta \rightarrow 0$ for $q \sim q_{\max }$ as in Fig. 11b (rather than $q$ fixed). In this limit, both $q_{\max }$ and the functional form for $\gamma$ in terms of $q / q_{\max }$ are different than in the magnetic case. We do not have a simple closed-form expression for this limit in the electric case.

Summarizing our results for all types of instability (electric and magnetic) for the planar distribution (1.1), we have found that there are two polarizations of instability for $\boldsymbol{q}$ within 45 degrees of the $z$ axis and one polarization otherwise. The most unstable modes are those with $\boldsymbol{q}$ close to or along the $z$ axis.

16 This instability corresponds to poles in $\Delta_{G}$ of Ref. [17] in the $\xi \rightarrow+\infty$ limit.

${ }^{17}$ By re-arranging terms algebraically and squaring, one can convert this into a quintic equation in $\eta^{2}$, a subset of whose roots are the solutions to (3.23). 


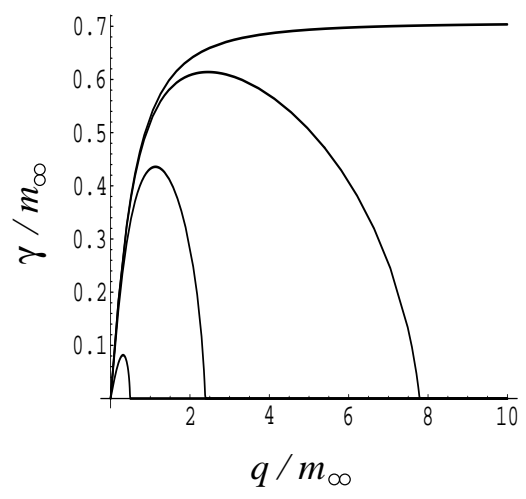

(a)

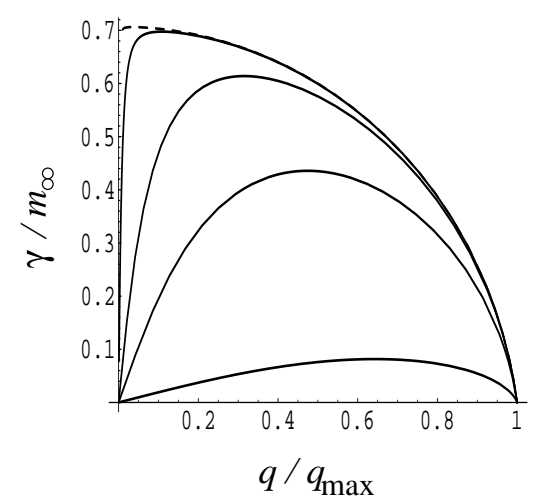

(b)

FIG. 11: The electric instability growth rate vs. $q$ for the planar distribution for various values of $\theta$. From the top down, the values are $\sin \theta=0.01,0.1,0.3,0.65$. The small dashed line at the top of (b) shows the $\sin \theta \rightarrow 0$ limit. There are no solutions for $\sin \theta>1 / \sqrt{2}$.

\section{NON-ABELIAN EXAMPLE OF SATURATING THE INSTABILITY}

We now give a concrete example of one particular way in which non-Abelian interactions between soft fields can halt the continuing growth of the amplitude of an unstable mode. Specifically, we will show that there exist static non-linear wave solutions to the soft field equations of motion which, in the linear approximation, would correspond to unstable modes. The soft field amplitudes will be of order $q / g$, as in (2.25). We will assume that the soft modes of interest have momentum $q$ much smaller than the momentum $p$ of typical hard particles, so that $g A / p \ll 1$. This means that the interactions of soft fields with each other must be treated non-perturbatively, but the interaction of soft fields with individual hard particles is perturbative. We can therefore ignore non-linear interactions induced by the hard particles and focus just on the perturbative self-energy $\Pi$ they induce for the soft fields. The relevant soft field equations are then the non-Abelian Maxwell equations with $j^{\mu}$ given as before by the perturbative self-energy:

$$
D_{\nu} F^{\mu \nu} \simeq \Pi^{\mu \nu} A_{\nu}
$$

where $F^{\mu \nu}$ is the full non-Abelian field strength and $D$ is the covariant derivative $\partial-i g A$ in the adjoint representation. The only assumption we will make about the hard particle distribution is that it is axi-symmetric about the $z$ axis so that (4.1) also has this symmetry.

\section{A. A solution}

Consider any $\mathrm{SU}(2)$ subgroup of the gauge group. Within this subgroup, we take the group structure constants to be the $\mathrm{SU}(2)$ ones $f^{a b c}=\epsilon^{a b c}$. Working in Lorentz gauge $\left(\partial_{\mu} A^{\mu}=0\right)$, our non-Abelian Maxwell equation is then

$$
\square A^{\mu a}+g \epsilon^{a b c} \partial_{\nu}\left(A^{\nu b} A^{\mu c}\right)+g^{2} \epsilon^{b a c} \epsilon^{c d e} A_{\nu}^{b} A^{\nu d} A^{\mu e}=\Pi^{\mu \nu} A_{\nu}^{a} .
$$

We will look for solutions with the ansatz

$$
A_{\mu}^{a}(\boldsymbol{x})= \begin{cases}\mathcal{A} R_{a \mu}(q z), & a=1 \text { or } 2, \text { and } \mu=1 \text { or } 2 \\ 0 & \text { otherwise }\end{cases}
$$


where $a$ is the adjoint color index and $R_{i j}(\theta)$ is the $2 \times 2$ rotation matrix

$$
R_{i j}(\theta)=\left(\begin{array}{rr}
\cos \theta & \sin \theta \\
-\sin \theta & \cos \theta
\end{array}\right) .
$$

The derivative in the second term of (4.2a) then vanishes. Using $R_{b i} R_{d i}=\delta_{b d}$, one then obtains

$$
\left(-q^{2}-2 g^{2} \mathcal{A}^{2}\right) \mathcal{A} R_{a \nu}(q z)=\Pi_{\perp}\left(0, q \boldsymbol{e}_{z}\right) \mathcal{A} R_{a \nu}(q z),
$$

where $\Pi_{\perp} \equiv \Pi^{x x}=\Pi^{y y}$. We have a solution if

$$
\mathcal{A}^{2}=-\frac{1}{2 g^{2}}\left[q^{2}+\Pi_{\perp}\left(0, q \boldsymbol{e}_{z}\right)\right]
$$

is positive. That will occur exactly when $q^{2}+\Pi_{\perp}\left(0, q \boldsymbol{e}_{z}\right)<0$, which is precisely Condition 1 of Sec. II B for a magnetic instability associated with small-amplitude waves with the same $\boldsymbol{q}=q \boldsymbol{e}_{z}$. Note that in our static non-linear solution, $\mathcal{A} \propto 1 / g$ and so is non-perturbatively large.

The energy density stored in the soft field is $\sim q^{2} \mathcal{A}^{2}$. Since $\mathcal{A} \sim q / g$ and $q \sim m_{\infty}$, the energy density in the soft field is $\sim m_{\infty}^{4} / g^{2}$. This is the same energy density we would obtain if all potentially unstable modes (of order half of all modes with $q<m_{\infty}$ ) carried occupation number $\sim 1 / g^{2}$. The latter is the situation we actually expect, when the instability saturates.

\section{B. A simple analog in scalar theory}

To motivate the ansatz (4.3), it is instructive to note that there are analogous static solutions of simple $\phi^{4}$ theory of a complex scalar $\phi$ with a Mexican hat potential

$$
V(\phi)=-\mu^{2}|\phi|^{2}+\frac{1}{2} \lambda|\phi|^{4}
$$

Here the equation of motion is

$$
\square \phi-\lambda|\phi|^{2} \phi=-\mu^{2} \phi
$$

and $-\mu^{2}$ plays the role of the destabilizing negative $\Pi$. By taking the ansatz

$$
\phi(\boldsymbol{x})=\mathcal{P} e^{i q z}
$$

where $e^{i \theta}$ is like the rotation matrix $R(\theta)$, one obtains

$$
\mathcal{P}^{2}=-\frac{1}{\lambda}\left[q^{2}-\mu^{2}\right]
$$

which gives a static solution for $q<\mu$. These $q$ 's correspond to what would be the unstable modes in a small fluctuation analysis about $\phi=0$.

The simplifications attending the use of a "helical" ansatz that rotates with $\theta=q z$, like the two ansatzes above, were also used in Ref. [32] to find a special case of non-linear solutions in the (qualitatively different) Abelian gauge theory problem. 
As far as we know, the scalar solution, like the non-Abelian one preceding it, is not relevant to any realistic physical situations. ${ }^{18}$ In typical applications where unstable fluctuations are growing, one will have an entire spectrum of different growing modes which will interact with each other non-linearly once they grow large enough. In the scalar problem, we would not expect a random spectrum of initial small fluctuations about $\phi=0$ to grow into a solution like (4.9). Instead, we expect this system to equilibrate, dissipating its energy into small random fluctuations about a minimum of the potential energy. Similarly, though our non-Abelian solution gives an example of how non-Abelian interactions can saturate the growth of instabilities, we expect the actual situation to be much more complicated.

As a final note on how things can be very different when only a limited number of modes are involved, consider a different ansatz we could have considered for the gauge theory problem that only considers modes with a single (adjoint) color:

$$
A_{\mu}^{a}(\boldsymbol{x})=\mathcal{A}(\boldsymbol{x}) \delta^{a 1} \varepsilon_{\mu} .
$$

With this ansatz, the non-Abelian non-linear equations (4.3) reduce to the Abelian linear equations (2.7). In this unnaturally idealized situation, the growth of instabilities would not be halted by soft interactions but, just like the Abelian case, would continue to grow until there were non-perturbative effects on the hard particles. We suspect that such a configuration in a non-Abelian setting would be unstable to the formation of large fields in other color directions, but we have not shown this explicitly.

\section{CONCLUSION}

We have seen that QCD plasma instabilities play an important role in the idealized theoretical limit of high energies and small coupling constants and drastically modify the bottom-up thermalization scenario. We have also seen reasons why the saturation of these instabilities should be qualitatively different from the Abelian case. An important goal for future work is to understand what replaces the original bottom-up scenario and determine in detail how these instabilities parametrically affect the rate of equilibration.

\section{Acknowledgments}

We would like to thank Larry Yaffe and Eugene Kolomeisky for useful conversations. This work was supported, in part, by the U.S. Department of Energy under Grant No. DEFG02-97ER41027, and by a McGill University startup grant.

\section{APPENDIX A: SUFFICIENT CONDITIONS FOR INSTABILITY}

In order to analyze the existence of instabilities, it will be convenient, initially, to phrase the discussion solely in terms of $\Pi^{i j}$. Note that $\Pi^{00}$ and $\Pi^{0 i}$ can be converted using the

${ }^{18}$ For $q>\mu$, one can alternatively find non-static solutions of the scalar theory of the form $\phi(\boldsymbol{x}, t)=\mathcal{P} e^{i \omega t}$. As far as we know, these are not useful for anything either. A broader class of related solutions for the quark-gluon plasmas has been investigated for stable, equilibrium plasmas in Ref. [36]. 
the Ward identity (2.9). We will follow Mrówczyński and others by rewriting the linearized Maxwell equation (2.7) in the form ${ }^{19}$

$$
D^{i j}(\omega, \boldsymbol{q}) E^{j} \equiv\left[\left(-\omega^{2}+q^{2}\right) \delta^{i j}-q^{i} q^{j}+\Pi^{i j}(\omega, \boldsymbol{q})\right] E^{j}=0 .
$$

One quick way to obtain this is to take the time derivative of (2.7) in $A^{0}=0$ gauge with $\mu=i$. The question of the existence of instabilities becomes whether $D^{i j}(\omega, \boldsymbol{q})$ ever has a zero eigenvalue for some $\omega$ with $\operatorname{Im} \omega>0$.

\section{Condition 1}

In searching for solutions to the dispersion relation with complex $\omega$, it will be convenient to drop the $i \epsilon$ prescription in the self-energy (2.8) and simply absorb it into the value of $\omega$ :

$$
\Pi^{i j}(\omega, \boldsymbol{q})=e^{2} \int_{\boldsymbol{p}} \frac{\partial f(\boldsymbol{p})}{\partial p^{k}}\left[-v^{i} \delta^{k j}+\frac{v^{i} v^{j} q^{k}}{-\omega+\boldsymbol{v} \cdot \boldsymbol{q}}\right] .
$$

Changing integration variables $\boldsymbol{p} \rightarrow-\boldsymbol{p}$ in this expression then implies $\Pi^{i j}(\omega)=\Pi^{i j}(-\omega)$. Complex conjugation then yields ${ }^{20}$

$$
\Pi^{i j}(\omega)=\left[\Pi^{i j}\left(-\omega^{*}\right)\right]^{*}
$$

In particular, consider pure positive imaginary $\omega$, and write $\omega=i \gamma$ with $\gamma$ real. Then (A3) implies $\Pi^{i j}(i \gamma)$ is a real matrix. Since it is also a symmetric matrix, it therefore has real eigenvalues.

The expression (A2) for $\Pi^{i j}(\omega, q)$ is bounded in magnitude as $\omega \rightarrow i \infty$. In this limit, $D^{i j}$ given by (A1) then becomes

$$
D^{i j}(\omega, \boldsymbol{q}) \rightarrow-\omega^{2} \delta^{i j}=\gamma^{2} \delta^{i j}
$$

with eigenvalues becoming identical and going to $+\infty$ as $\gamma \rightarrow \infty$. On the other hand, for $\gamma=0$

$$
D^{i j}(0, \boldsymbol{q})=q^{2} \delta^{i j}-q^{i} q^{j}+\Pi^{i j}(0, \boldsymbol{q})
$$

which is the $3 \times 3$ matrix of Condition 1' of Sec. II B. Suppose $D^{i j}(0, \boldsymbol{q})$ has one or more negative eigenvalues, such as shown in Fig. 12a. By continuity of the real eigenvalues of $D^{i j}(0, i \gamma)$ as $\gamma$ is varied from 0 to $\infty$, there must then be some positive $\gamma$ for which $D^{i j}(0, i \gamma)$ has a zero eigenvalue, which corresponds to an unstable solution. And there must exist one such solution for each negative eigenvalue of $D^{i j}(0, \boldsymbol{q})$. This proves Condition 1', which is equivalent to Condition 1.

19 This equation is gauge invariant in Abelian theory. Gauge invariance in non-Abelian theory is not manifest simply because we are studying instabilities by linearizing in the fields. The equation is gauge invariant up to non-linear terms that have been neglected.

${ }^{20}$ For $\omega=\omega_{0}+i \epsilon$ with $\omega_{0}$ real, this implies $\Pi_{\text {ret }}^{i j}\left(\omega_{0}\right)=-\Pi_{\text {adv }}^{i j}\left(-\omega_{0}\right)=-\left[\Pi_{\text {ret }}^{i j}\left(-\omega_{0}\right)\right]^{*}$, where "ret" and "adv" denote the self-energies for retarded and advanced Green's functions. 


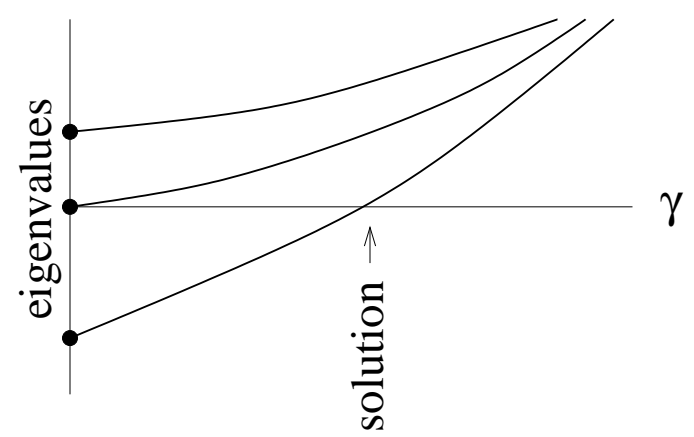

(a)

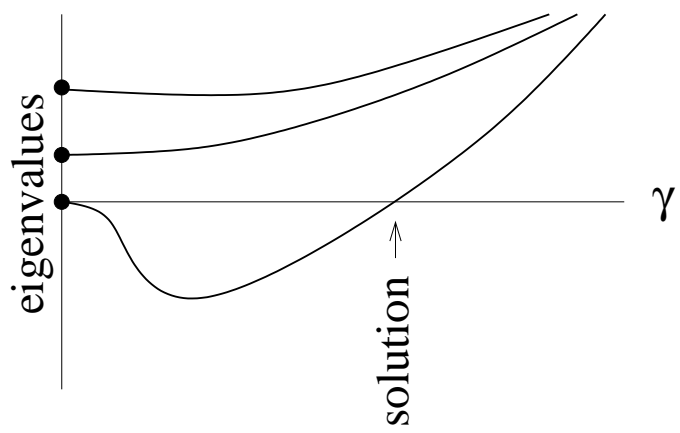

(b)

FIG. 12: Examples of the continuity of eigenvalues of $D^{i j}(i \gamma, \boldsymbol{q})$ with $\gamma$, depicting (a) one magnetic instability [Condition 1], and (b) one electric instability [Condition 2].

\section{Condition 2}

There is always a zero eigenvalue of $D^{i j}(0, \boldsymbol{q})$ associated with the longitudinal polarization $\hat{\boldsymbol{q}}$, and the above argument does not tell us whether there is a $\gamma>0$ solution $D^{i j}(i \gamma, \boldsymbol{q})=0$ associated with the continuous evolution of this eigenmode as $\gamma$ varies from 0 to $\infty$. However, suppose we knew that the corresponding eigenvalue was negative for very small positive $\gamma$. The previous eigenvalue continuity argument would then guarantee a corresponding instability, as depicted in Fig. 12b.

Let us therefore investigate the small eigenvalue $\lambda$ of $D^{i j}(i \gamma, \boldsymbol{q})$ for arbitrarily small and positive $\gamma$. Let $\top$ represent the two directions transverse to $\boldsymbol{q}$. Making use of the Ward identity (2.9), the matrix $D^{i j}(\omega, \boldsymbol{q})$ for small $\omega$ has the form

$$
D(\omega, \boldsymbol{q})=\left(\begin{array}{cc}
-\omega^{2}+\Pi^{\hat{\hat{q}}}(\omega, \boldsymbol{q}) & \Pi^{\hat{\hat{T}}}(\omega, \boldsymbol{q}) \\
\Pi^{\top \hat{q}}(\omega, \boldsymbol{q}) & q^{2}+\Pi^{\top \top}(\omega, \boldsymbol{q})
\end{array}\right) \simeq\left(\begin{array}{cc}
-\omega^{2}+\frac{\omega^{2}}{q^{2}} \Pi^{00}(0, \boldsymbol{q}) & \frac{\omega}{q} \Pi^{0 \top}(0, \boldsymbol{q}) \\
\frac{\omega}{q} \Pi^{\top 0}(0, \boldsymbol{q}) & q^{2}+\Pi^{\top \top}(0, \boldsymbol{q})
\end{array}\right),
$$

where $\Pi(0, \boldsymbol{q})$ above should be understood (as in the main text) as the retarded self-energy at zero frequency, $\Pi(i \epsilon, \boldsymbol{q})$. In the limit of small $\omega$, the small eigenvalue of such a matrix is

$$
\lambda \simeq \frac{\omega^{2}}{q^{2}}\left[-q^{2}+\Pi^{00}-\Pi^{0 \top}\left(q^{2}+\Pi^{\top \top}\right)^{-1} \Pi^{0 \top}\right]_{\omega=i \epsilon},
$$

where $\left[q^{2}+\Pi^{\top \top}\right]^{-1}$ is the inverse of the $2 \times 2$ matrix $q^{2}+\Pi^{\top \top}$. Condition 2 of Sec. II B is just the condition that the eigenvalue (A7) be negative for small imaginary $\omega$.

\section{Relation to Nyquist analysis}

In textbooks [25, 26], it is more usual to derive the Penrose criteria from a Nyquist analysis rather than to use continuity arguments as above. However, the usual analysis becomes more complicated than textbook examples for general distributions $f(\boldsymbol{p})$ and general directions $\hat{\boldsymbol{q}}$, and we find the continuity argument simpler to implement for reasons we now explain.

The textbook derivations work whenever one can use symmetry to reduce the matrix dispersion relation (A1) to single-component dispersion relations. If one is looking for solutions 


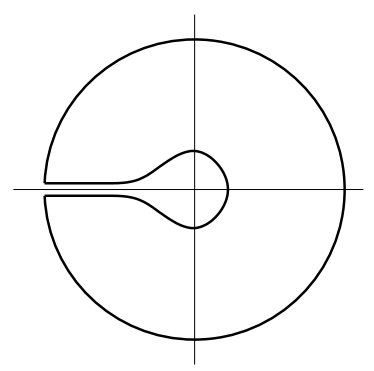

(a)

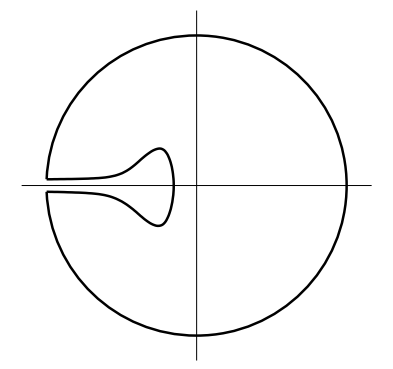

(b)

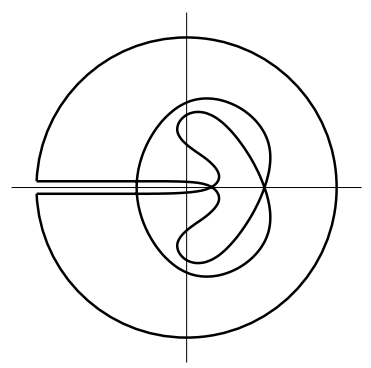

(c)

FIG. 13: Examples of Nyquist contours $D(C)$ in the complex plane. The large outer circle represents the image of the semi-circle at infinity, and the rest is the image of $\Re+i \epsilon$. [Diagram (c) is not just a fanciful example. It is also the form of the Nyquist diagram associated with the function $\eta^{-2}$ times the left-hand side of (3.23) for certain values of $\boldsymbol{q}$ and $\sin \theta$ that give instability. The single winding denotes a single associated unstable mode.]

to a single component relation of the form

$$
D(\omega, \boldsymbol{q}) \equiv-\omega^{2}+q^{2}+\Pi(\omega, \boldsymbol{q})=0
$$

and if $\Pi$ is an analytic function of $\omega$ in the upper half plane, then one can count the number of zeros of $D(\omega)$ for fixed $\boldsymbol{q}$ (which gives the number of unstable solutions) using the Residue Theorem as

$$
N=\oint_{C} \frac{d \omega}{2 \pi i} \frac{1}{D} \frac{d D}{d \omega}=\oint \frac{d(\ln D)}{2 \pi i}
$$

where the contour $C$ encloses the upper half $\omega$ plane by (i) running infinitesimally above the real axis (which we will denote $\Re+i \epsilon$ ) and then (ii) closing in a semi-circle at infinity. The last form above shows that $N$ is just the winding number about the origin of the image $D(C)$ of the curve $C$. For $|\omega| \rightarrow \infty$, the self-energy given by (2.18) is bounded, and so $D(\omega) \rightarrow-\omega^{2}$, so that the semi-circle at infinity maps to a circle at infinity that almost closes. Fig. 13 shows various examples of possible images $D(C)$. The winding number in all these cases is even if $\Pi(i \epsilon)$ is positive and odd otherwise, for the following reasons. Count the winding number by counting the number of times (and sense) that $D(C)$ crosses the negative real axis. The curves $D(C)$ are symmetric with respect to complex conjugation because the conjugation property (A3) of the self-energy implies $\Pi(x+i \epsilon)=\Pi^{*}(-x+i \epsilon)$ for real $x$, so that the image of the half-line infinitesimally above the positive real axis must be the conjugate of that above the negative real axis. This conjugation property means that, except at $\omega=i \epsilon$, any crossing of the real axis by $D(C)$ must be at a point where the real axis is crossed twice. As a result, all the crossings except for $\omega=i \epsilon$ do not affect whether the winding number is odd or even, which is solely determined by $\Pi(i \epsilon)$. If $\Pi(i \epsilon)$ is odd, one knows that the winding number cannot be zero, which proves the sufficiency of the Penrose criterion.

The difficulty with the above analysis in the multi-component case is that it relies on $D(\omega)$ being analytic in the upper half plane. Suppose that in the general multi-component case of (A1) one wants to develop three independent instability conditions, corresponding to the three eigenvalues of the matrix $D$. One could imagine algebraically solving for the eigenvalues to get three one-component equations. However, solving the characteristic equation to obtain 
eigenvalues introduces root singularities and branch cuts. If any of these appeared in the upper-half plane, it would destroy the analyticity assumption of the Nyquist analysis. It may be possible to construct an analysis of this sort, but we found it easier to avoid the issue altogether.

One may forgo finding independent conditions and simply calculate the total number of unstable solutions by doing a Nyquist analysis with the single equation $\operatorname{det} D=0$. However, there are not useful corresponding Penrose criteria because the sign of $\operatorname{det} D(i \epsilon)$ would depend on whether there were an even or odd number of unstable modes.

For cases where the problem can be reduced to one-component equations like (A8), one often sees the Penrose criteria used as necessary as well as sufficient conditions for instability. This happens, for example, in situations where one can argue that the sign of $\operatorname{Im} \Pi(x+i \epsilon)$ is the same as the sign of $x$ for real $x \neq 0$, so that the only place $D(\Re+i \epsilon)$ can cross the real axis is for $\omega=i \epsilon$. Then the winding number is either exactly zero or one, as in Figs. $13 \mathrm{a}$ and $\mathrm{b}$, depending on the sign of $\Pi(i \epsilon)$. For example, consider the case with $\hat{\boldsymbol{q}}$ in the $z$ direction, $f(\boldsymbol{p})=F\left(p_{z}\right) H\left(p_{\perp}\right)$, and polarization in the $x$ direction. Taking the imaginary part of (2.8) for real $\omega$ gives

$$
\operatorname{Im} \Pi^{\varepsilon \varepsilon}(\omega, \boldsymbol{q})=e^{2} \pi \int_{\boldsymbol{p}} \frac{\partial f}{\partial p_{z}}\left(v_{x}\right)^{2} \delta\left(-\eta+v_{z}\right),
$$

where $\eta \equiv \omega /|\boldsymbol{q}|$. The sign of this result is then just the sign of $\partial F / \partial p_{z}$ evaluated at $v_{z}=\eta$. If $F\left(p_{z}\right)$ is a monotonically decreasing function of $\left|p_{z}\right|$, then the sign of the result will indeed be the sign of $\omega$, and the Penrose condition would be necessary as well as sufficient for that mode.

Unfortunately, matters are more complicated when $\hat{\boldsymbol{q}}$ does not point in a symmetry direction. Suppose again that $f(\boldsymbol{p})$ had the form $F\left(p_{z}\right) H\left(p_{\perp}\right)$, with both $F\left(p_{z}\right)$ and $H\left(p_{\perp}\right)$ monotonically decreasing functions of $\left|p_{z}\right|$ and $p_{\perp}$. One could easily make a similar argument as above if $\operatorname{Im} \Pi^{i j}(\omega, \boldsymbol{q})$ were a positive definite matrix for positive real $\omega$. One can check positive definiteness by checking $\varepsilon^{i} \operatorname{Im} \Pi^{i j} \varepsilon^{j}>0$ for arbitrary real spatial polarizations $\varepsilon$. Eq. (13) gives a result of the form

$$
\varepsilon_{i} \operatorname{Im}\left[\Pi^{i j}(\omega, \boldsymbol{q})\right] \varepsilon_{j}=\int_{\boldsymbol{p}}[\text { positive }] \delta(-\eta+\boldsymbol{v} \cdot \hat{\boldsymbol{q}}) \hat{\boldsymbol{q}} \cdot \nabla_{\boldsymbol{p}} f .
$$

Unfortunately, $\hat{\boldsymbol{q}} \cdot \boldsymbol{\nabla}_{\boldsymbol{p}} f$ is not necessarily positive when $\boldsymbol{v} \cdot \hat{\boldsymbol{q}}$ is positive, as shown in Fig. 14. We are unaware of a general proof that the Penrose criteria are necessary conditions for instability in such cases.

\section{APPENDIX B: ANISOTROPY CREATES INSTABILITY (CONDITION 1-B)}

First, we will show that non-vanishing $\Pi^{i j}(0, \hat{\boldsymbol{q}})$ implies magnetic instabilities. Start by considering the trace of the spatial part of (2.12), and then spatially average that trace over the direction of $\hat{\boldsymbol{q}}$ :

$$
\left\langle\Pi^{i i}(0, \hat{\boldsymbol{q}})\right\rangle_{\hat{\boldsymbol{q}}}=e^{2} \int_{\boldsymbol{p}} \frac{\partial f(\boldsymbol{p})}{\partial p^{k}}\left[-v^{k}+v^{2}\left\langle\frac{\hat{q}^{k}}{\boldsymbol{v} \cdot \hat{\boldsymbol{q}}-i \epsilon}\right\rangle_{\hat{\boldsymbol{q}}}\right] .
$$




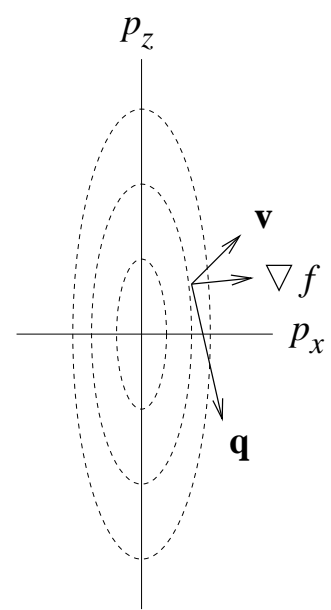

FIG. 14: An example (shown in the $x z$ plane) of $f(\boldsymbol{p}), \boldsymbol{p}$ and $\boldsymbol{q}$ for which $\boldsymbol{v} \cdot \boldsymbol{\nabla} f$ and $\boldsymbol{v} \cdot \boldsymbol{q}$ do not have the same sign. The ellipses indicate curves of constant $f$.

It is easy to see that

$$
\left\langle\frac{\hat{\boldsymbol{q}}}{\boldsymbol{v} \cdot \hat{\boldsymbol{q}}-i \epsilon}\right\rangle_{\hat{\boldsymbol{q}}}=\frac{\boldsymbol{v}}{v^{2}}
$$

because the left-hand side (i) gives unity when dotted with $\boldsymbol{v}$, and (ii) must be proportional to $\boldsymbol{v}$ by rotational invariance. We therefore obtain

$$
\left\langle\Pi^{i i}(0, \hat{\boldsymbol{q}})\right\rangle_{\hat{\boldsymbol{q}}}=0
$$

But the trace $\Pi^{i i}$ is the sum of the eigenvalues of $\Pi^{i j}$. What we have shown is that the eigenvalues of $\Pi^{i j}(0, \hat{\boldsymbol{q}})$, averaged over all eigenvalues and all directions of $\hat{\boldsymbol{q}}$, average to zero. Therefore, either all eigenvalues in all directions are identically zero, or some eigenvalue in some direction must be negative (implying an instability). In particular it is a necessary condition for stability that the trace $\Pi^{i i}(0, \hat{\boldsymbol{q}})$ should vanish for all $\hat{\boldsymbol{q}}$.

Now we show that anisotropic $\mathcal{M}(\hat{\boldsymbol{p}})$, defined by (2.13), implies non-vanishing trace $\Pi^{i i}(0, \hat{\boldsymbol{q}})$ for some $\hat{\boldsymbol{q}}$ and therefore implies the existence of instabilities. Taking the trace and setting $\omega=0$ in (2.18), we have

$$
\Pi^{i i}(0, \boldsymbol{q})=e^{2} \int_{\boldsymbol{p}} f(\boldsymbol{p}) \frac{v}{p}\left[1+\frac{1}{(\hat{\boldsymbol{p}} \cdot \hat{\boldsymbol{q}}-i \epsilon)^{2}}\right] .
$$

Note that the factor in brackets does not depend on the magnitudes $p$ and $v$ of $\boldsymbol{p}$ and $\boldsymbol{v}$ but only on their common direction $\hat{\boldsymbol{p}}$. Performing the integral over $p$ gives a factor of $\mathcal{M}(\hat{\boldsymbol{p}})$ defined by (2.13):

$$
\Pi^{i i}(0, \hat{\boldsymbol{q}})=\left\langle\mathcal{M}(\hat{\boldsymbol{p}})\left[1+\frac{1}{(\hat{\boldsymbol{p}} \cdot \hat{\boldsymbol{q}}-i \epsilon)^{2}}\right]\right\rangle_{\hat{\boldsymbol{p}}} .
$$

Now decompose $\mathcal{M}$ into spherical harmonics:

$$
\mathcal{M}(\hat{\boldsymbol{p}})=\sum_{l m} a_{l m} Y_{l m}(\hat{\boldsymbol{p}})
$$


By rotational invariance, Eq. (B5) will generate an angular dependence proportional to $Y_{l m}(\hat{\boldsymbol{q}})$ for each term proportional to $Y_{l m}(\hat{\boldsymbol{p}})$. So

$$
\Pi^{i i}(0, \hat{\boldsymbol{q}})=\sum_{l m} \kappa_{l} a_{l m} Y_{l m}(\hat{\boldsymbol{q}})
$$

where

$$
\kappa_{l} \equiv 4 \pi\left\langle Y_{l m}^{*}(\hat{\boldsymbol{q}})\left[1+\frac{1}{(\hat{\boldsymbol{p}} \cdot \hat{\boldsymbol{q}}-i \epsilon)^{2}}\right] Y_{l m}(\hat{\boldsymbol{p}})\right\rangle_{\hat{\boldsymbol{p}}, \hat{\boldsymbol{q}}}
$$

does not depend on $m$, again because of rotational invariance.

The only way that (B5) can vanish for all $\hat{\boldsymbol{q}}$ is if all of the $a_{l m} \kappa_{l}$ vanish. If $\mathcal{M}(\hat{\boldsymbol{p}})$ is anisotropic, then one of the $a_{l m}$ must be non-zero for $l>0$, and it must be an even $l$ because of our universal assumption that $f(\boldsymbol{p})$ is parity symmetric. If we knew that $\kappa_{l} \neq 0$ for all even $l>0$, we would then know that $\Pi^{i i}(0, \hat{\boldsymbol{q}})$ cannot vanish for anisotropic $\mathcal{M}(\hat{\boldsymbol{p}})$. Therefore we turn to the evaluation of $\kappa_{l}$.

To evaluate $\kappa_{l}$, first choose $m=0$ in (B8). Next, use Wigner $D$ functions to re-express the spherical harmonic $Y_{l 0}(\hat{\boldsymbol{p}})$ defined with respect to a fixed $z$ axis in terms of spherical harmonics $Y_{l m^{\prime}}^{(\hat{\boldsymbol{q}})}(\hat{\boldsymbol{p}})$ defined with respect to the direction $\hat{\boldsymbol{q}}$ :

$$
Y_{l m}(\hat{\boldsymbol{p}})=\sum_{m^{\prime}} D_{m m^{\prime}}^{l}(\hat{\boldsymbol{q}}) Y_{l m^{\prime}}^{(\hat{\boldsymbol{q}})}(\hat{\boldsymbol{p}})
$$

Let $\theta$ be the angle between $\hat{\boldsymbol{p}}$ and $\hat{\boldsymbol{q}}$ and note that

$$
\left\langle\left[1+\frac{1}{(\cos \theta-i \epsilon)^{2}}\right] Y_{l m^{\prime}}^{(\hat{\boldsymbol{q}})}(\hat{\boldsymbol{p}})\right\rangle_{\hat{\boldsymbol{p}}}
$$

vanishes unless $m^{\prime}=0$. So

$$
\begin{aligned}
\left\langle\left[1+\frac{1}{(\hat{\boldsymbol{p}} \cdot \hat{\boldsymbol{q}}-i \epsilon)^{2}}\right] Y_{l 0}(\hat{\boldsymbol{p}})\right\rangle_{\hat{\boldsymbol{p}}}=D_{00}^{l}(\hat{\boldsymbol{q}}) & \left\langle\left[1+\frac{1}{(\hat{\boldsymbol{p}} \cdot \hat{\boldsymbol{q}}-i \epsilon)^{2}}\right] Y_{l 0}^{(\hat{\boldsymbol{q}})}(\hat{\boldsymbol{p}})\right\rangle_{\hat{\boldsymbol{p}}} \\
& =Y_{l 0}(\hat{\boldsymbol{q}})\left\langle\left[1+\frac{1}{(\cos \theta-i \epsilon)^{2}}\right] P_{l}(\cos \theta)\right\rangle_{\hat{\boldsymbol{p}}}
\end{aligned}
$$

which gives

$$
\kappa_{l}=\frac{1}{2} \int_{-1}^{1} d x\left[1+\frac{1}{(x-i \epsilon)^{2}}\right] P_{l}(x),
$$

where $P_{l}(x)$ is the $l$-th Legendre polynomial. This integral gives ${ }^{21}$

$$
\kappa_{l}=\delta_{l 0}-\frac{(-)^{\frac{l}{2}} \sqrt{\pi}\left(\frac{l}{2}\right) !}{\Gamma\left(\frac{l}{2}+\frac{1}{2}\right)}=\delta_{l 0}-\frac{(-)^{\frac{l}{2}} l ! !}{(l-1) ! !}
$$

for even $l$, which indeed does not vanish for any even $l>0$.

${ }^{21}$ This may be derived by an appropriate analytic continuation of Eq. (7.126.1) of Ref. [37]. 
This completes the proof that anisotropic $\mathcal{M}(\hat{\boldsymbol{p}})$ implies magnetic instabilities. What about the converse? Suppose $\mathcal{M}(\hat{\boldsymbol{p}})$ is isotropic. In the ultra-relativistic limit $(v=1)$, we can perform the radial $p$ integral of the expression (2.18) for the self-energy to write

$$
\Pi^{i j}(\omega, \boldsymbol{q})=\left\langle\mathcal{M}(\hat{\boldsymbol{p}})\left[\delta^{i j}-\frac{\hat{q}^{i} \hat{p}^{j}+\hat{q}^{j} \hat{p}^{i}}{-\eta+\hat{\boldsymbol{p}} \cdot \hat{\boldsymbol{q}}-i \epsilon}+\frac{\left(-\eta^{2}+1\right) \hat{p}^{i} \hat{p}^{j}}{(-\eta+\hat{\boldsymbol{p}} \cdot \hat{\boldsymbol{q}}-i \epsilon)^{2}}\right]\right\rangle_{\hat{\boldsymbol{p}}}
$$

with $\eta \equiv \omega / q$. If $\mathcal{M}(\hat{\boldsymbol{p}})$ is isotropic, then we can factor it out to give

$$
\Pi^{i j}(\omega, \boldsymbol{q})=m_{\infty}^{2}\left\langle\delta^{i j}-\frac{\hat{q}^{i} \hat{p}^{j}+\hat{q}^{j} \hat{p}^{i}}{-\eta+\hat{\boldsymbol{p}} \cdot \hat{\boldsymbol{q}}-i \epsilon}+\frac{\left(-\eta^{2}+1\right) \hat{p}^{i} \hat{p}^{j}}{(-\eta+\hat{\boldsymbol{p}} \cdot \hat{\boldsymbol{q}}-i \epsilon)^{2}}\right\rangle_{\hat{\boldsymbol{p}}} .
$$

All of the details of the distribution $f(\boldsymbol{p})$ have factored out into the single normalization constant $m_{\infty}^{2}$. Except for this normalization, there is no difference between the general case of isotropic $\mathcal{M}(\hat{\boldsymbol{p}})$ and the specific case of equilibrium distributions. In equilibrium, there is no instability, and so there cannot be one for any isotropic $\mathcal{M}(\hat{\boldsymbol{p}})$. (One may check this explicitly using the standard results for the equilibrium self-energy [38, 39].)

\section{APPENDIX C: INSTABILITY OF THE ULTRA-RELATIVISTIC LINE MO- MENTUM DISTRIBUTION}

In the bulk of this paper, we have focused on instabilities associated with the planar (extreme oblate) distribution of (1.1). It is also interesting to study the other extreme of the linear (extreme prolate) distribution of (1.2):

$$
f(\boldsymbol{p}, \boldsymbol{x})=F\left(p_{z}\right) \delta^{(2)}\left(\boldsymbol{p}_{\perp}\right) .
$$

Our standard assumption of parity symmetry is here that $F\left(-p_{z}\right)=F\left(p_{z}\right)$. We will specialize to the ultra-relativistic case, where we will find that the distribution $(\mathrm{C} 1)$ is always associated with electric instabilities (in the sense of Condition 2 of Sec. II B).

Consider directions of $\hat{\boldsymbol{q}}$ that do not lie in the $x y$ plane, i.e. $\theta \neq \pi / 2$. From (2.18), we get the self-energy

$$
\Pi^{i j}(\omega, \boldsymbol{q})=e^{2} \int_{\boldsymbol{p}} \frac{f(\boldsymbol{p})}{p}\left[\delta^{i j}-\frac{\hat{q}^{i} \hat{p}^{j}+\hat{q}^{j} \hat{p}^{i}}{-\eta+\hat{\boldsymbol{p}} \cdot \hat{\boldsymbol{q}}-i \epsilon}+\frac{\left(-\eta^{2}+1\right) \hat{p}^{i} \hat{p}^{j}}{(-\eta+\hat{\boldsymbol{p}} \cdot \hat{\boldsymbol{q}}-i \epsilon)^{2}}\right] .
$$

Naively, one may use the $\delta$ function in the distribution $(\mathrm{C} 1)$ to do all but the $p_{z}$ integral. Summing over the cases $p_{z}<0$ and $p_{z}>0$ (corresponding to $\hat{p}_{z}= \pm 1$ for this distribution),

$$
\Pi^{i j}(\omega, \boldsymbol{q})=\frac{1}{2} m_{\infty}^{2} \sum_{ \pm}\left[\delta^{i j} \mp \frac{\hat{q}^{i} \delta^{j z}+\hat{q}^{j} \delta^{i z}}{-\eta \pm \cos \theta-i \epsilon}+\frac{\left(-\eta^{2}+1\right) \delta^{i z} \delta^{j z}}{(-\eta \pm \cos \theta-i \epsilon)^{2}}\right]
$$

with $m_{\infty}$ naively given by $(2.14)$ as

$$
m_{\infty}^{2}=e^{2} \int_{\boldsymbol{p}} \frac{f(\boldsymbol{p})}{p}=\frac{2 e^{2}}{(2 \pi)^{3}} \int_{0}^{\infty} d p_{z} \frac{F\left(p_{z}\right)}{p_{z}} .
$$

The preceding is slightly naive if $F(0) \neq 0$, since then the integral (C4) has a logarithmic, small $p_{z}$ divergence. In physical situations, the $\delta^{(2)}\left(\boldsymbol{p}_{\perp}\right)$ in $(\mathrm{C} 1)$ would have a small 
width $\Delta p_{\perp}$, and the small $p_{z}$ divergence would cut off when $p_{z} \sim \Delta p_{\perp}$. Then $m_{\infty}^{2}$ will be finite, growing proportional to $F(0) \ln \left[(\Delta \theta)^{-1}\right]$ as $\Delta p_{\perp} \rightarrow 0$, where $\Delta \theta \sim p_{\perp} / \bar{p}$ and $\bar{p}$ is a characteristic momentum scale of $F\left(p_{z}\right)$. If we are only interested in results to leading order in this logarithm (which come from $p_{z} \gg \Delta p$ ), then the equation (C3) is fine. ${ }^{22}$ It should then be understood that this and future equations in this section are only accurate up to relative corrections of order $1 / \ln \left[(\Delta \theta)^{-1}\right]$.

As usual, let $\boldsymbol{q}$ lie in the $x z$ plane. The $y$ direction is then an eigen-direction of $\Pi^{i j}$, and (C3) gives

$$
\Pi^{y y}(\omega, \boldsymbol{q})=m_{\infty}^{2} .
$$

There is no instability associated with this polarization. The remaining directions give

$$
\begin{aligned}
& \Pi^{x x}(\omega, \boldsymbol{q})=m_{\infty}^{2} \\
& \Pi^{x z}(\omega, \boldsymbol{q})=m_{\infty}^{2} \frac{\sin \theta \cos \theta}{\eta^{2}-\cos ^{2} \theta} \\
& \Pi^{z z}(\omega, \boldsymbol{q})=m_{\infty}^{2} \frac{\sin ^{2} \theta\left(\eta^{2}+\cos ^{2} \theta\right)}{\left(\eta^{2}-\cos ^{2} \theta\right)^{2}}
\end{aligned}
$$

or equivalently

$$
\begin{aligned}
\Pi^{\hat{\boldsymbol{q}} \hat{\boldsymbol{q}}}(\omega, \boldsymbol{q}) & =\eta^{2} \Pi^{00}(\omega, \boldsymbol{q})=m_{\infty}^{2} \eta^{2} \sin ^{2} \theta \frac{\eta^{2}+\cos ^{2} \theta}{\left(\eta^{2}-\cos ^{2} \theta\right)^{2}} \\
\Pi^{\hat{\boldsymbol{q}} \hat{\boldsymbol{n}}}(\omega, \boldsymbol{q}) & =\eta \Pi^{0 \hat{\boldsymbol{n}}}(\omega, \boldsymbol{q})=m_{\infty}^{2} \eta^{2} \sin \theta \cos \theta \frac{\left(-2+\eta^{2}+\cos ^{2} \theta\right)}{\left(\eta^{2}-\cos ^{2} \theta\right)^{2}} \\
\Pi^{\hat{\boldsymbol{n}} \hat{\boldsymbol{n}}}(\omega, \boldsymbol{q}) & =m_{\infty}^{2} \frac{\left(-1+\eta^{2}\right)^{2} \cos ^{2} \theta+\eta^{2} \sin ^{4} \theta}{\left(\eta^{2}-\cos ^{2} \theta\right)^{2}}
\end{aligned}
$$

In all cases, we have absorbed the $i \epsilon$ prescription into $\eta$ so that $\eta$ now stands for $\eta+i \epsilon$.

We shall look for all solutions to the dispersion relations in a moment. It is worth noting first that $\Pi^{\hat{\boldsymbol{n}} \hat{\boldsymbol{n}}}(0, \hat{\boldsymbol{q}})=m_{\infty}^{2} / \cos ^{2} \theta$, so that Condition 1 of Sec. II B for a magnetic instability is not satisfied for $\theta \neq \pi / 2$. This makes qualitative sense because, for $\hat{\boldsymbol{q}}$ not in the $x y$ plane, the distribution $(\mathrm{C} 1)$ does not give any trapped particles. [The singularity of $\Pi^{\hat{\boldsymbol{n}} \hat{\boldsymbol{n}}}(0, \hat{\boldsymbol{q}})$ for $\theta=\pi / 2$ is a reflection of the zero width of the transverse $\delta$ function in the distribution (C1) and will be cut off by a small width $\Delta p_{\perp}$. From Condition 1-b, we know there must be a magnetic instability associated with $\theta=\pi / 2$.]

In contrast, $\Pi^{00}(0, \hat{\boldsymbol{q}})=m_{\infty}^{2} \tan ^{2} \theta$ and $\Pi^{0 \hat{\boldsymbol{n}}}(0, \hat{\boldsymbol{q}})=0$ do indicate the existence of an electric instability by Condition 2 when

$$
q<q_{\max }=m_{\infty} \tan \theta
$$

Note that there is no instability for $\sin \theta=0$, which is when $\boldsymbol{q}$ points in the same direction as the particle motion. As discussed in Sec. II C, the lack of an electric instability in this

${ }^{22}$ The non-relativistic case is very different. Doing a similar computation of $\Pi^{00}(0, \hat{\boldsymbol{q}})$ in the non-relativistic case, one finds a linear rather than logarithmic small $p_{z}$ divergence when $F(0) \neq 0$. As a result, the $\delta$-function approximation in the distribution $(\mathrm{C} 1)$ is never valid for calculating $\Pi^{00}(0, \hat{\boldsymbol{q}})$ in the nonrelativistic case when $F(0) \neq 0$. 
case is a reflection of the fact that the speed of ultra-relativistic particles cannot change from $v=1$.

The nice thing about the distribution (C1) is that we can in fact completely solve the dispersion relation in simple closed form. The $y y$ dispersion relation $-\omega^{2}+q^{2}+\Pi^{y y}(\omega, \boldsymbol{q})=0$, with (C5), just gives the two propagating solutions

$$
\omega^{2}=q^{2}+m_{\infty}^{2}
$$

For polarizations in the $x z$ plane, we turn to the $x z$ subspace of the form (A1) of the dispersion relation. There will be a solution when $\operatorname{det} D$ (taken over this subspace) vanishes. Using the results (C6) for $\Pi$, this condition $D^{x x} D^{z z}-\left(D^{x z}\right)^{2}=0$ [or equivalently $D^{\hat{\boldsymbol{q}} \hat{\boldsymbol{q}}} D^{\hat{\boldsymbol{n}} \hat{\boldsymbol{n}}}-$ $\left(D^{\hat{\boldsymbol{q}} \hat{\boldsymbol{n}}}\right)^{2}=0$ ] can be simplified to

$$
Q^{2}\left(\eta^{2}-\cos ^{2} \theta\right)^{2}\left(\eta^{2} Q^{2}-Q^{2}-1\right)+\sin ^{2} \theta=0,
$$

where we use the dimensionless variable $Q \equiv q / m_{\infty}$. This condition can be factorized into

$$
\left(\eta^{2}-c^{2}-Q^{-2}\right)\left[Q^{2} \eta^{4}-Q^{2} \eta^{2}\left(1+c^{2}\right)+\left(c^{2} Q^{2}-s^{2}\right)\right]=0
$$

where $c \equiv \cos \theta$ and $s \equiv \sin \theta$. There are three corresponding solutions for $\omega^{2}$, in addition to (C8), which are

$$
\omega^{2}=q^{2} \cos ^{2} \theta+m_{\infty}^{2}
$$

and

$$
\omega^{2}=\frac{1}{2}\left[q^{2}\left(1+\cos ^{2} \theta\right) \pm q \sin \theta \sqrt{q^{2} \sin ^{2} \theta+4 m_{\infty}^{2}}\right] .
$$

The lower sign in the last solution gives an instability $\left(\omega^{2}<0\right)$ when $q<q_{\max }$, as given by (C7). This is the only instability of this distribution for $\theta \neq \pi / 2$.

\section{APPENDIX D: DISPERSION RELATION FOR PLANAR DISTRIBUTION}

In this appendix, we briefly describe the derivation of the dispersion relation (3.23) used to study electric instabilities for the planar distribution (1.1). We look for solutions to the matrix dispersion relation (A1) for polarizations in the $x z$ plane by requiring the determinant of $D$ in that subspace to vanish:

$$
0=D^{x x} D^{z z}-\left(D^{x z}\right)^{2}=\left(-\omega^{2}+q_{z}^{2}+\Pi^{x x}\right)\left(-\omega^{2}+q_{x}^{2}+\Pi^{z z}\right)-\left(-q_{x} q_{z}+\Pi^{x z}\right)^{2},
$$

or equivalently

$$
0=D^{\hat{\boldsymbol{q}} \hat{\boldsymbol{q}}} D^{\hat{\boldsymbol{n}} \hat{\boldsymbol{n}}}-\left(D^{\hat{\boldsymbol{q}} \hat{\boldsymbol{n}}}\right)^{2}=\left(-\omega^{2}+\Pi^{\hat{\boldsymbol{q}} \hat{\boldsymbol{q}}}\right)\left(-\omega^{2}+q^{2}+\Pi^{\hat{\boldsymbol{n}} \hat{\boldsymbol{n}}}\right)-\left(\Pi^{\hat{\boldsymbol{q}} \hat{\boldsymbol{n}}}\right)^{2} .
$$

Starting from (3.6) and performing the integral over the angle $\phi$ of $\hat{\boldsymbol{p}}$ in the $x y$ plane, one obtains

$$
\begin{aligned}
& \Pi^{z z}=m_{\infty}^{2} \\
& \Pi^{x z}=\frac{c}{s}\left[\left(1-\frac{s^{2}}{\eta^{2}}\right)^{-1 / 2}-1\right] m_{\infty}^{2} \\
& \Pi^{x x}=\frac{1}{s^{2}}\left[c^{2}\left(1-\frac{s^{2}}{\eta^{2}}\right)^{-3 / 2}-\left(2 c^{2}-\eta^{2}\right)\left(1-\frac{s^{2}}{\eta^{2}}\right)^{-1 / 2}+c^{2}-\eta^{2}\right] m_{\infty}^{2},
\end{aligned}
$$


where $s \equiv \sin \theta$ and $c \equiv \cos \theta$. Combining these expressions gives a result for (D1) that is $s^{-2}$ times the right-hand side of (3.23).

In the limit $\sin \theta \rightarrow 0$, the matrix $D$ becomes diagonal with

$$
\begin{aligned}
& D^{z z} \rightarrow m_{\infty}^{2}-\eta^{2} q^{2} \\
& D^{x x} \rightarrow q^{2}\left(1-\eta^{2}\right)+\frac{m_{\infty}^{2}}{2}\left(\frac{1}{\eta^{2}}+1\right)
\end{aligned}
$$

In this limit, the unstable mode, given by (3.24), corresponds to $\boldsymbol{E}$ polarized in the $x$ direction. Note, in contrast, that the Penrose condition that predicted the existence of this unstable mode via Condition 2 was associated with the $\hat{\boldsymbol{q}}$ eigen-direction of $\Pi^{i j}(i \epsilon, \boldsymbol{q}$ ) (see Appendix A 2), which for $\sin \theta \rightarrow 0$ is the $z$ direction. This just reflects the fact that the eigen-directions can change as one varies $\omega$ from $i \epsilon$ to the location $i \gamma$ of the actual unstable solution.

[1] S. Mrówczyński, "Stream instabilities of the quark-gluon plasma," Phys. Lett. B 214, 587 (1988).

[2] S. Mrówczyński, "Plasma instability at the initial stage of ultrarelativistic heavy ion collisions," Phys. Lett. B 314, 118 (1993).

[3] S. Mrówczyński, "Color collective effects at the early stage of ultrarelativistic heavy ion collisions," Phys. Rev. C 49, 2191 (1994).

[4] S. Mrówczyński, "Color filamentation in ultrarelativistic heavy-ion collisions," Phys. Lett. B 393, 26 (1997) [hep-ph/9606442].

[5] S. Mrówczyński and M. H. Thoma, "Hard loop approach to anisotropic systems," Phys. Rev. D 62, 036011 (2000) [hep-ph/0001164].

[6] J. Randrup and S. Mrówczyński, "Chromodynamic Weibel instabilities in relativistic nuclear collisions," nucl-th/0303021.

[7] U. W. Heinz, "Quark-qluon transport theory," Nucl. Phys. A 418, 603C (1984).

[8] Y. E. Pokrovsky and A. V. Selikhov, "Filamentation in a quark-gluon plasma," JETP Lett. 47, 12 (1988) [Pisma Zh. Eksp. Teor. Fiz. 47, 11 (1988)].

[9] Y. E. Pokrovsky and A. V. Selikhov, "Filamentation in quark plasma at finite temperatures," Sov. J. Nucl. Phys. 52, 146 (1990) [Yad. Fiz. 52, 229 (1990)].

[10] Y. E. Pokrovsky and A. V. Selikhov, "Filamentation in the quark-gluon plasma At finite temperatures," Sov. J. Nucl. Phys. 52, 385 (1990) [Yad. Fiz. 52, 605 (1990)].

[11] O. P. Pavlenko, "Filamentation instability of hot quark-gluon plasma with hard jet," Sov. J. Nucl. Phys. 55, 1243 (1992) [Yad. Fiz. 55, 2239 (1992)].

[12] R. Baier, A. H. Mueller, D. Schiff and D. T. Son, 'Bottom-up' thermalization in heavy ion collisions," Phys. Lett. B 502, 51 (2001) [hep-ph/0009237].

[13] L. V. Gribov, E. M. Levin and M. G. Ryskin, "Semihard Processes In QCD," Phys. Rept. 100, 1 (1983).

[14] A. H. Mueller and J. w. Qiu, "Gluon Recombination And Shadowing At Small Values Of x," Nucl. Phys. B 268, 427 (1986).

[15] J. P. Blaizot and A. H. Mueller, "The Early Stage Of Ultrarelativistic Heavy Ion Collisions," Nucl. Phys. B 289, 847 (1987). 
[16] L. D. McLerran and R. Venugopalan, "Computing quark and gluon distribution functions for very large nuclei," Phys. Rev. D 49, 2233 (1994) [hep-ph/9309289]; "Green's functions in the color field of a large nucleus," Phys. Rev. D 50, 2225 (1994) [hep-ph/9402335].

[17] P. Romatschke and M. Strickland, "Screening and antiscreening in anisotropic quark gluon plasma," hep-ph/0304092.

[18] T.-Y. B. Yang, Y. Gallant, J. Arons, A. B. Langdon, "Weibel instability in relativistically hot magnetized electron-positron plasma," Phys. Fluids B 5, 3369 (1993).

[19] E. S. Weibel, "Spontaneously growing transverse waves in a plasma due to an anisotropic velocity distribution," Phys. Rev. Lett. 2, 83 (1959).

[20] T. S. Biro, B. Muller and X. N. Wang, "Color screening in relativistic heavy ion collisions," Phys. Lett. B 283, 171 (1992).

[21] J. Bjoraker and R. Venugopalan, "From colored glass condensate to gluon plasma: Equilibration in high energy heavy ion collisions," Phys. Rev. C 63, 024609 (2001) [hep-ph/0008294].

[22] G. C. Nayak, A. Dumitru, L. D. McLerran and W. Greiner, "Equilibration of the gluon-minijet plasma at RHIC and LHC," Nucl. Phys. A 687, 457 (2001) [hep-ph/0001202].

[23] U. Heinz, "Kinetic theory for nonabelian plasmas," Phys. Rev. Lett. 51, 351 (1983); "Quarkqluon transport theory. Part 1. The classical theory," Annals Phys. 161, 48 (1985); "Quarkqluon transport theory. Part 2. Color response and color correlations in a quark-gluon plasma," ibid. 168, 148 (1986).

[24] M. C. Birse, C. W. Kao and G. C. Nayak, "Magnetic screening effects in anisotropic QED and QCD plasmas," hep-ph/0304209.

[25] N. A. Krall and A. W. Trivelpiece, Principles of Plasma Physics (McGraw-Hill, 1973).

[26] R. C. Davidson, "Kinetic waves and instabilites in a uniform plasma" in Basic Plasma Physics I (vol. 1 of Handbook of Plasma Physics), eds. A. A. Galeev and R. N. Sudan (North-Holland, 1984).

[27] P. Arnold, G. D. Moore and L. G. Yaffe, "Effective kinetic theory for high temperature gauge theories," JHEP 0301, 030 (2003) [hep-ph/0209353].

[28] O. Buneman, "Instability, turbulence, and conductivity in current-carrying plasma," Phys. Rev. Lett. 1, 8 (1958).

[29] D. Bohm and E. P. Gross, "Theory of plasma oscillations. A. Origin of medium-like behavior," Phys. Rev. 75, 1851 (1949).

[30] I. B. Bernstein, J. M. Greene, and M. D. Kruskal, "Exact nonlinear plasma oscillations," Phys. Rev. 108, 546 (1957).

[31] R. J. Goldston and P.H. Rutherford, Introduction to Plasma Physics (IOP Publishing, 1995).

[32] R. L. Berger and R. C. Davidson, "Equilibrium and stability of large-amplitude magnetic Bernstein-Greene-Kruskal waves," Phys. Fluids 15, 2327 (1972).

[33] R. C. Davidson, D. A. Hammer, U. Haber, and C. E. Wagner, "Nonlinear development of electromagnetic instabilities in anisotropic plasmas," Phys. Fluids 15, 317 (1972).

[34] T.-Y. B. Yang, J. Arons, A. B. Langdon, "Evolution of the Weibel instability in relativistically hot electron-positron beams," Phys. Plasmas 1, 3059 (1994).

[35] F. Califano, N. Attico, F. Pegoraro, G. Bertin, and S. V. Bulanov, "Fast formation of magnetic islands in a plasma in the presence of counterstreaming electrons," Phys. Rev. Lett. 86, 5293 (2001).

[36] J. P. Blaizot and E. Iancu, "Non-Abelian plane waves in the quark-gluon plasma," Phys. Lett. B 326, 138 (1994) [hep-ph/9401323].

[37] I. S. Gradshteyn and I. M. Ryzhik, Table of Integrals, Series, and Products, 4th edition 
(Academic Press, 1980).

[38] O. K. Kalashnikov and V. V. Klimov, Sov. J. Nucl. Phys. 31, 699 (1980) [Yad. Fiz. 31, 1357 (1980)].

[39] H. A. Weldon, Phys. Rev. D26, 1394 (1982). 


\section{Erratum: QCD plasma instabilities and bottom-up thermalization [JHEP 08 (2003) 002]}

Peter Arnold, Jonathan Lenaghan, and Guy D. Moore

In the last sentence of the paragraph containing (3.11), $q \simeq m_{\infty}^{2}\left(\frac{2}{3} \sin ^{2} \theta\right)^{1 / 4}$ should instead read $q \simeq m_{\infty}\left[2 /\left(3 \sin ^{2} \theta\right)\right]^{1 / 4}$.

Before eq. (4.1), there is a misleadingly general statement about when one can ignore non-linear interactions induced by hard particles. They can be ignored for gauge fields which only depend on a single coordinate, such as $A=A(z)$ as considered subsequently, but not in general. See, for example, Ref. [36], as well as Ref. [40] below.

Eq. (4.1) should have an overall minus sign on the right-hand side. In eq. (4.2), the $\epsilon^{b a c}$ in the $g^{2}$ term should be $\epsilon^{a b c}$. Furthermore, the left-hand side should of eq. (4.2) should have an additional term $+g \epsilon^{a b c} A_{\nu}^{b}\left(\partial^{\nu} A^{c \mu}-\partial^{\mu} A^{c \nu}\right)$. The last error is not merely typographic but was due to simultaneous cosmic ray events in the brains of the three authors. As a result, the solution presented in section 4.1 is not in fact a solution to the equations of motion. This does not affect the rest of the paper. Actual static (but unstable) solutions analogous to the non-linear solutions of Ref. [36] may be found in Ref. [40].

The last paragraph in section 4.1 is misleadingly general. It applies only to cases where the typical momenta of unstable modes are of order $m_{\infty}$. For the approximately planar hard-particle distributions considered in most of the paper, the typical unstable momentum scale is instead of order $q_{\max } \gg m_{\infty}$, where $q_{\max }$ is given by eq. (3.13).

[40] P. Arnold and J. Lenaghan, "The abelianization of QCD plasma instabilities," hep$\mathrm{ph} / 0408052$. 